\title{
Oscillation Criteria for Some New Generalized Emden-Fowler Dynamic Equations on Time Scales
}

\author{
Haidong Liu ${ }^{1}$ and Puchen Liu ${ }^{2}$ \\ ${ }^{1}$ School of Mathematical Sciences, Qufu Normal University, Shandong 273165, China \\ ${ }^{2}$ Department of Mathematics, University of Houston, Houston, TX 77204, USA
}

Correspondence should be addressed to Haidong Liu; tomlhd983@163.com

Received 4 September 2012; Revised 8 January 2013; Accepted 11 January 2013

Academic Editor: Patricia J. Y. Wong

Copyright (C) 2013 H. Liu and P. Liu. This is an open access article distributed under the Creative Commons Attribution License, which permits unrestricted use, distribution, and reproduction in any medium, provided the original work is properly cited.

By means of novel analytical techniques, we have established several new oscillation criteria for the generalized Emden-Fowler dynamic equation on a time scale $\mathbb{T}$, that is, $\left(r(t)\left|Z^{\Delta}(t)\right|^{\alpha-1} Z^{\Delta}(t)\right)^{\Delta}+f(t, x(\delta(t)))=0$, with respect to the case $\int_{t_{0}}^{\infty} r^{-1 / \alpha}(s) \Delta s=\infty$ and the case $\int_{t_{0}}^{\infty} r^{-1 / \alpha}(s) \Delta s<\infty$, where $Z(t)=x(t)+p(t) x(\tau(t)), \alpha$ is a constant, $|f(t, u)| \geqslant q(t)\left|u^{\beta}\right|, \beta$ is a constant satisfying $\alpha \geqslant \beta>0$, and $r, p$, and $q$ are real valued right-dense continuous nonnegative functions defined on $\mathbb{T}$. Noting the parameter value $\alpha$ probably unequal to $\beta$, our equation factually includes the existing models as special cases; our results are more general and have wider adaptive range than others' work in the literature.

\section{Introduction}

In the past two decades, the theory of time scales proposed by Hilger [1] in 1990 has received extensive attention because of its advantage to unify continuous model and discrete model into one case under the scholars' investigation. Numerous authors have considered many aspects of this new theory. Many of those results focus on oscillation and nonoscillation of some equations on time scales. Reader can refer to articles [2-25] and there references cited therein.

In this paper, we consider the oscillatory behavior of the solutions of second-order generalized Emden-Fowler dynamic equation of the form

$$
\left(r(t)\left|Z^{\Delta}(t)\right|^{\alpha-1} Z^{\Delta}(t)\right)^{\Delta}+f(t, x(\delta(t)))=0, \quad t \in \mathbb{T}, t \geqslant t_{0},
$$

with $Z(t)=x(t)+p(t) x(\tau(t))$, parameter constant $\alpha$, and conditions $\left(\mathrm{H}_{1}\right)-\left(\mathrm{H}_{6}\right)$ :

$\left(\mathrm{H}_{1}\right) \mathbb{T}$ is a time scale which is unbounded above. $\left[t_{0}, \infty\right)_{\mathbb{T}}:=\left[t_{0}, \infty\right) \cap \mathbb{T}$, where $t_{0} \in \mathbb{T}$ with $t_{0}>0$, $C_{\mathrm{rd}}(\mathbb{T}, \mathbb{S})$ denotes the collection of all functions $f$ : $\mathbb{T} \rightarrow \mathbb{S}$ which are right-dense continuous on $\mathbb{T}$;

$$
\begin{aligned}
& \left(\mathrm{H}_{2}\right) r(t) \in C_{\mathrm{rd}}(\mathbb{T},(0, \infty)), R(t):=\int_{t_{0}}^{t} r^{-1 / \alpha}(s) \Delta s ; \\
& \left(\mathrm{H}_{3}\right) p(t) \in C_{\mathrm{rd}}(\mathbb{T},[0,1]) ; \\
& \left(\mathrm{H}_{4}\right) \tau(t) \in C_{\mathrm{rd}}(\mathbb{T}, \mathbb{T}), \tau(t) \leqslant t, \text { for } t \in \mathbb{T}, \lim _{t \rightarrow \infty} \tau(t)= \\
& \quad \infty, \delta(t) \in C_{\mathrm{rd}}(\mathbb{T}, \mathbb{T}), \delta(t) \leqslant t, \text { for } t \in \mathbb{T}, \lim _{t \rightarrow \infty} \delta(t)= \\
& \quad \infty ;
\end{aligned}
$$

$\left(\mathrm{H}_{5}\right) \delta^{\Delta}(t)>0$ is right-dense continuous on $\mathbb{T}$, and $\delta(\sigma(t))=\sigma(\delta(t))$ for all $t \in \mathbb{T}$, where $\sigma(t)$ is the forward jump operator on $\mathbb{T}$;

$\left(\mathrm{H}_{6}\right) f(t, u) \in C(\mathbb{T} \times \mathbb{R}, \mathbb{R})$ is a continuous function such that $u f(t, u)>0$, for all $u \neq 0$ and there exists a positive right-dense continuous function $q(t)$ defined on $\mathbb{T}$ such that $|f(t, u)| \geqslant q(t)\left|u^{\beta}\right|$ for all $t \in \mathbb{T}$ and for all $u \in \mathbb{R}$, where $\beta$ is a constant satisfying $\alpha \geqslant \beta>0$.

As a solution of (1), we mean a function $x(t)$ such that $x(t)+p(t) x(\tau(t)) \in C_{\mathrm{rd}}^{1}\left(t_{x}, \infty\right)_{\mathbb{T}}$ and $r(t) \mid[x(t)+$ $p(t) x(\tau(t))]\left.^{\Delta}\right|^{\alpha-1}[x(t)+p(t) x(\tau(t))]^{\Delta} \in C_{\mathrm{rd}}^{1}\left(t_{x}, \infty\right)_{\mathbb{T}}, t_{x} \geqslant t_{0}$ and satisfying (1) for all $t \geqslant t_{x}$, where $C_{\mathrm{rd}}^{1}\left(t_{x}, \infty\right)_{\mathbb{T}}$ denotes the set of right-dense continuously $\Delta$-differentiable functions on $\left(t_{x}, \infty\right)_{\mathbb{T}}$. In the sequel, we restrict our attention to those solutions of (1) which exist on the half-line $\left[t_{x}, \infty\right)_{\mathbb{T}}$ and satisfy $\sup \{|x(t)|: t>\widetilde{T}\}>0$ for any $\widetilde{T} \geqslant t_{x}$. We say that 
a nontrivial solution of (1) is oscillatory if it has arbitrary large zeros, otherwise we say that it is nonoscillatory. We say that (1) is oscillatory if all its solutions are oscillatory.

Among researchers in the oscillation of functional equations with time scales, Agarwal et al. [2] studied a special case of (1), which is

$$
\begin{aligned}
& \left(r(t)\left(\left[y(t)+p(t) y\left(t-\tau_{0}\right)\right]^{\Delta}\right)^{\gamma}\right)^{\Delta} \\
& \quad+f\left(t, y\left(t-\delta_{0}\right)\right)=0, \quad t \in \mathbb{T}, t \geqslant t_{0},
\end{aligned}
$$

where

$$
\begin{aligned}
& |f(t, u)| \geqslant q(t)|u|^{\gamma}, \\
& \int_{t_{0}}^{\infty} r^{-1 / \gamma}(s) \Delta s=\infty,
\end{aligned}
$$

$\tau_{0}$ and $\delta_{0}$ are positive constants and $\gamma>0$ is a quotient of odd positive integers. They got some oscillation criteria of (2) for the case when $\gamma>0$ under the condition $r^{\Delta}(t) \geqslant 0$, and the case when $\gamma \geqslant 1$ under the condition $\mu(t)>0$. Subsequently, for the case when $\gamma \geqslant 1$ is an odd positive integer, Saker [7] did not require the conditions $r^{\Delta}(t) \geqslant 0$ and $\mu(t)>0$ and obtained some new oscillation results for (2) under the conditions (3).

Very Recently, in [10-13], Saker et al. have considered the oscillation of several equations with time scales. For example in paper [13], the author is concerned with the quasilinear equation of the form:

$$
\left(p(t)\left([y(t)+r(t) y(\tau(t))]^{\Delta}\right)^{\gamma}\right)^{\Delta}+f(t, y(\delta(t)))=0,
$$

where $|f(t, u)| \geqslant q(t)\left|u^{\beta}\right|, \gamma>0$, and $\beta>0$ are ratios of odd positive integers.

However the value range of the equation parameters in our work is wider than those in $[2,7,10-13]$ and the equation itself is also different from those in [2, 7, 10-13]. In fact, our approach in constructing the criteria is different from those of Saker and his coauthors' work.

For (2) with $\gamma \geqslant 1$ being a quotient of odd positive integers and without the restrictive conditions $r^{\Delta}(t) \geqslant 0$ and without $\mu(t)>0$, Wu et al. [21] obtained several oscillation criteria for the equation:

$$
\begin{array}{r}
\left(r(t)\left([y(t)+p(t) y(\tau(t))]^{\Delta}\right)^{\gamma}\right)^{\Delta}+f(t, y(\delta(t)))=0, \\
t \in \mathbb{T}, t \geqslant t_{0},
\end{array}
$$

under the conditions (3).

Chen [25] investigated the following second-order Emden-Fowler neutral delay dynamic equation

$$
\begin{array}{r}
\left(r(t)\left|x^{\Delta}(t)\right|^{\gamma-1} x^{\Delta}(t)\right)^{\Delta}+f(t, y(\delta(t)))=0, \\
t \in \mathbb{T}, t \geqslant t_{0},
\end{array}
$$

with $x(t)=y(t)+p(t) y(\tau(t))$, under the conditions (3). He obtained some oscillation criteria when $\gamma>0$ is a constant and without assuming the conditions $r^{\Delta}(t) \geqslant 0$ and $\mu(t)>0$.

All the above results cannot apply to our model (1) since our model (1) is more general than (2), (6) and those in [10-13], and the function $f(t, u)$ in $(1)$ satisfies $\left(\mathrm{H}_{6}\right)$ which makes our model (1) distinguished from all the existing cases. To the best of our knowledge, nothing is known regarding the necessary and sufficient conditions for the qualitative behavior of (1) with $\alpha \neq \beta$ in $\left(\mathrm{H}_{6}\right)$ on time scales.

In this paper, even if $\alpha \neq \beta$ in $\left(\mathrm{H}_{6}\right)$ and there is no assumptions $r^{\Delta}(t) \geqslant 0$ and $\mu(t)>0$, we have established several new oscillation criteria of (1) for the both cases

$$
\begin{aligned}
& \lim _{t \rightarrow \infty} \int_{t_{0}}^{t} r^{-1 / \alpha}(s) \Delta s=\infty, \\
& \lim _{t \rightarrow \infty} \int_{t_{0}}^{t} r^{-1 / \alpha}(s) \Delta s<\infty .
\end{aligned}
$$

Factually, we have employed new analytical techniques to present and construct our criteria in Section 3 after reciting two useful lemmas in Section 2. Our results have extended and unified a number of other existing results and handled the cases which are not covered by current criteria. Finally, in Section 4 two examples are demonstrated to illustrate the efficiency of our work with relevant remark.

\section{Some Lemmas}

Lemma 1 (see $[25]$ ). Suppose that $\left(H_{5}\right)$ holds. Let $x: \mathbb{T} \rightarrow \mathbb{R}$. If $x^{\Delta}$ exists for all sufficiently large $t \in \mathbb{T}$, then $(x(\delta(t)))^{\Delta}=$ $x^{\Delta}(\delta(t)) \delta^{\Delta}(t)$ for all sufficiently large $t \in \mathbb{T}$.

Lemma 2 (Bohner and Peterson [26, Theorem 1.90]). Assume that $x(t)$ is $\Delta$-differentiable and eventually positive or eventually negative, then

$$
\left(x^{\alpha}(t)\right)^{\Delta}=\alpha\left\{\int_{0}^{1}[(1-h) x(t)+h x(\sigma(t))]^{\alpha-1} d h\right\} x^{\Delta}(t) .
$$

Lemma 3 (see [27]). Let $\Psi(u)=a u-b u^{(\lambda+1) / \lambda}$, where $a, b, \lambda$ are constants, $a \geqslant 0, b>0, \lambda>0$, and $u \in[0, \infty)$. Then $\Psi(u)$ attains its maximum value on $[0, \infty)$ at $u=u^{*}:=(a \lambda / b(\lambda+$ 1) $)^{\lambda}$, and

$$
\max _{u \in[0, \infty)} \Psi(u)=\Psi\left(u^{*}\right)=\frac{\lambda^{\lambda}}{(\lambda+1)^{\lambda+1}} \frac{a^{\lambda+1}}{b^{\lambda}}
$$

\section{Main Results}

The case

$$
\lim _{t \rightarrow \infty} \int_{t_{0}}^{t} r^{-1 / \alpha}(s) \Delta s=\infty
$$


Theorem 4. Assume that $\left(H_{1}\right)-\left(H_{6}\right)$ and (7) hold. If there exists a function $\xi(t) \in C_{\mathrm{rd}}^{1}(\mathbb{T},(0, \infty))$ such that for any positive number $M$,

$$
\varlimsup_{t \rightarrow \infty} \int_{t_{0}}^{t}(\xi(s) \bar{p}(s)-Q(s)) \Delta s=\infty,
$$

where

$$
\begin{gathered}
\bar{p}(s)=q(s)[1-p(\delta(s))]^{\beta}, \\
Q(s)=\frac{\alpha^{\alpha} M(R(\sigma(s)))^{\alpha-\beta} r(\delta(s))\left(\left(\xi^{\Delta}(s)\right)_{+}\right)^{\alpha+1}}{(\alpha+1)^{\alpha+1} \beta^{\alpha} \xi^{\alpha}(s)\left(\delta^{\Delta}(s)\right)^{\alpha}}, \\
\left(\xi^{\Delta}(s)\right)_{+}:=\max \left\{\xi^{\Delta}(s), 0\right\},
\end{gathered}
$$

then (1) is oscillatory.

Proof. Suppose that (1) has a nonoscillatory solution $x(t)$, then there exists $T_{0} \geqslant t_{0}$ such that $x(t) \neq 0$ for all $t \geqslant T_{0}$. Without loss of generality, we assume that $x(t)>0, x(\tau(t))>$ 0 and $x(\delta(t))>0$ for $t \geqslant T_{0}$, because a similar analysis holds for $x(t)<0, x(\tau(t))<0$ and $x(\delta(t))<0$. Then the following are deduced from $(1),\left(\mathrm{H}_{3}\right)$, and $\left(\mathrm{H}_{6}\right)$ :

$$
\begin{gathered}
Z(t) \geqslant x(t)>0 \quad \text { for } t \geqslant T_{0}, \\
\left(r(t)\left|Z^{\Delta}(t)\right|^{\alpha-1} Z^{\Delta}(t)\right)^{\Delta} \leqslant 0, \quad t \geqslant T_{0} .
\end{gathered}
$$

Therefore $r(t)\left|Z^{\Delta}(t)\right|^{\alpha-1} Z^{\Delta}(t)$ is a nonincreasing function and $Z^{\Delta}(t)$ is eventually of one sign.

We claim that

$$
Z^{\Delta}(t)>0 \quad \text { or } \quad Z^{\Delta}(t)=0, \quad t \geqslant T_{0} .
$$

Otherwise, if there exists a $t_{1} \geqslant T_{0}$ such that $Z^{\Delta}(t)<0$ for $t \geqslant t_{1}$, then from (14), for some positive constant $K$, we have

$$
-r(t)\left(-Z^{\Delta}(t)\right)^{\alpha} \leqslant-K, \quad t \geqslant t_{1},
$$

that is,

$$
-Z^{\Delta}(t) \geqslant\left(\frac{K}{r(t)}\right)^{1 / \alpha}, \quad t \geqslant t_{1},
$$

integrating the above inequality from $t_{1}$ to $t$, we have

$$
Z(t) \leqslant Z\left(t_{1}\right)-K^{1 / \alpha}\left(R(t)-R\left(t_{1}\right)\right) .
$$

Letting $t \rightarrow \infty$, from (7), we get $\lim _{t \rightarrow \infty} Z(t)=-\infty$, which contradicts (14). Thus, we have proved (15).

We choose some $T_{1} \geqslant T_{0}$ such that $\delta(t) \geqslant T_{0}$ for $t \geqslant T_{1}$. Therefore from (14), (15), and the fact $\delta(t) \leqslant \sigma(t)$, we have that

$$
r(\sigma(t))\left(Z^{\Delta}(\sigma(t))\right)^{\alpha} \leqslant r(\delta(t))\left(Z^{\Delta}(\delta(t))\right)^{\alpha}, \quad t \geqslant T_{1} \text {, }
$$

which follows that

$$
Z^{\Delta}(\delta(t)) \geqslant Z^{\Delta}(\sigma(t))\left(\frac{r(\sigma(t))}{r(\delta(t))}\right)^{1 / \alpha}, \quad t \geqslant T_{1} .
$$

On the other hand, from (1), $\left(\mathrm{H}_{6}\right)$, and (15), we have

$$
\begin{aligned}
& \left(r(t)\left(Z^{\Delta}(t)\right)^{\alpha}\right)^{\Delta}+q(t)(Z(\delta(t))-p(\delta(t)) x(\tau(\delta(t))))^{\beta} \\
& \leqslant 0, \quad t \geqslant T_{1} .
\end{aligned}
$$

Noticing (15) and the fact $Z(t) \geqslant x(t)$, we get

$$
\left(r(t)\left(Z^{\Delta}(t)\right)^{\alpha}\right)^{\Delta}+\bar{p}(t) Z^{\beta}(\delta(t)) \leqslant 0, \quad t \geqslant T_{1},
$$

where $\bar{p}(t)=q(t)[1-p(\delta(t))]^{\beta}$.

Define

$$
w(t)=\xi(t) \frac{r(t)\left(Z^{\Delta}(t)\right)^{\alpha}}{Z^{\beta}(\delta(t))}, \quad \text { for } t \geqslant T_{1} .
$$

Obviously, $w(t)>0$. By (22), (23) and the product rule and the quotient rule, we obtain

$$
\begin{aligned}
w^{\Delta}(t)= & \frac{\xi(t)}{Z^{\beta}(\delta(t))}\left(r(t)\left(Z^{\Delta}(t)\right)^{\alpha}\right)^{\Delta}+r(\sigma(t))\left(Z^{\Delta}(\sigma(t))\right)^{\alpha} \\
& \times \frac{\xi^{\Delta}(t) Z^{\beta}(\delta(t))-\xi(t)\left(Z^{\beta}(\delta(t))\right)^{\Delta}}{Z^{\beta}(\delta(t)) Z^{\beta}(\delta(\sigma(t)))} \\
\leqslant & -\xi(t) \bar{p}(t)+\frac{\xi^{\Delta}(t)}{\xi(\sigma(t))} w(\sigma(t)) \\
& -\frac{r(\sigma(t))\left(Z^{\Delta}(\sigma(t))\right)^{\alpha} \xi(t)\left(Z^{\beta}(\delta(t))\right)^{\Delta}}{Z^{\beta}(\delta(t)) Z^{\beta}(\delta(\sigma(t)))} .
\end{aligned}
$$

Now we consider the following two cases.

Case 1. Let $\beta \geqslant 1$. By (15), Lemmas 1 and 2, we have

$$
\begin{aligned}
&\left(Z^{\beta}(\delta(t))\right)^{\Delta} \\
&=\beta\left\{\int_{0}^{1}[(1-h) Z(\delta(t))+h Z(\delta(\sigma(t)))]^{\beta-1} \mathrm{~d} h\right\} \\
& \\
& \quad \times(Z(\delta(t)))^{\Delta} \\
& \geqslant \beta(Z(\delta(t)))^{\beta-1} Z^{\Delta}(\delta(t)) \delta^{\Delta}(t) .
\end{aligned}
$$


From $\left(\mathrm{H}_{5}\right),(20),(23)-(25)$, and the fact that $Z(t)$ is nondecreasing, we obtain

$$
\begin{aligned}
& w^{\Delta}(t) \\
& \leqslant-\xi(t) \bar{p}(t)+\frac{\xi^{\Delta}(t)}{\xi(\sigma(t))} w(\sigma(t)) \\
& -\frac{r(\sigma(t))\left(Z^{\Delta}(\sigma(t))\right)^{\alpha} \xi(t) \beta(Z(\delta(t)))^{\beta-1} Z^{\Delta}(\delta(t)) \delta^{\Delta}(t)}{Z^{\beta}(\delta(t)) Z^{\beta}(\delta(\sigma(t)))} \\
& \leqslant-\xi(t) \bar{p}(t)+\frac{\xi^{\Delta}(t)}{\xi(\sigma(t))} w(\sigma(t)) \\
& -\frac{r(\sigma(t))\left(Z^{\Delta}(\sigma(t))\right)^{\alpha} \xi(t) \beta Z^{\Delta}(\delta(t)) \delta^{\Delta}(t)}{Z^{\beta+1}(\delta(\sigma(t)))} \\
& \leqslant-\xi(t) \bar{p}(t)+\frac{\xi^{\Delta}(t)}{\xi(\sigma(t))} w(\sigma(t)) \\
& -\frac{\beta \xi(t) r(\sigma(t))\left(Z^{\Delta}(\sigma(t))\right)^{\alpha+1} \delta^{\Delta}(t)}{Z^{\beta+1}(\delta(\sigma(t)))} \\
& \times\left(\frac{r(\sigma(t))}{r(\delta(t))}\right)^{1 / \alpha} \\
& =-\xi(t) \bar{p}(t)+\frac{\xi^{\Delta}(t)}{\xi(\sigma(t))} w(\sigma(t)) \\
& -\frac{\beta \xi(t) \delta^{\Delta}(t)}{(\xi(\sigma(t)))^{1+1 / \alpha}(Z(\delta(\sigma(t))))^{(\alpha-\beta) / \alpha}(r(\delta(t)))^{1 / \alpha}} \\
& \times w^{(\alpha+1) / \alpha}(\sigma(t)) \\
& =-\xi(t) \bar{p}(t)+\frac{\xi^{\Delta}(t)}{\xi(\sigma(t))} w(\sigma(t)) \\
& -\frac{\beta \xi(t) \delta^{\Delta}(t)}{(\xi(\sigma(t)))^{1+1 / \alpha}(Z(\sigma(t)))^{(\alpha-\beta) / \alpha}(r(\delta(t)))^{1 / \alpha}} \\
& \times w^{(\alpha+1) / \alpha}(\sigma(t)) \text {. }
\end{aligned}
$$

Case 2. Let $0<\beta<1$. By (15), Lemmas 1 and 2 , we get

$$
\begin{aligned}
&\left(Z^{\beta}(\delta(t))\right)^{\Delta} \\
&=\beta\left\{\int_{0}^{1}[(1-h) Z(\delta(t))+h Z(\delta(\sigma(t)))]^{\beta-1} \mathrm{~d} h\right\} \\
& \quad \times(Z(\delta(t)))^{\Delta} \\
& \geqslant \beta(Z(\delta(\sigma(t))))^{\beta-1} Z^{\Delta}(\delta(t)) \delta^{\Delta}(t) .
\end{aligned}
$$

From $\left(\mathrm{H}_{4}\right),\left(\mathrm{H}_{5}\right),(20),(23)-(25)$, and the fact that $Z(t)$ is nondecreasing, we have

$w^{\Delta}(t)$

$$
\begin{aligned}
& \leqslant-\xi(t) \bar{p}(t)+\frac{\xi^{\Delta}(t)}{\xi(\sigma(t))} w(\sigma(t)) \\
& -\frac{r(\sigma(t))\left(Z^{\Delta}(\sigma(t))\right)^{\alpha} \xi(t) \beta(Z(\delta(\sigma(t))))^{\beta-1} Z^{\Delta}(\delta(t)) \delta^{\Delta}(t)}{Z^{\beta}(\delta(t)) Z^{\beta}(\delta(\sigma(t)))} \\
& =-\xi(t) \bar{p}(t)+\frac{\xi^{\Delta}(t)}{\xi(\sigma(t))} w(\sigma(t)) \\
& -\frac{r(\sigma(t))\left(Z^{\Delta}(\sigma(t))\right)^{\alpha} \xi(t) \beta Z^{\Delta}(\delta(t)) \delta^{\Delta}(t)}{Z^{\beta+1}(\delta(\sigma(t)))} \\
& \leqslant-\xi(t) \bar{p}(t)+\frac{\xi^{\Delta}(t)}{\xi(\sigma(t))} w(\sigma(t)) \\
& -\frac{\beta \xi(t) r(\sigma(t))\left(Z^{\Delta}(\sigma(t))\right)^{\alpha+1} \delta^{\Delta}(t)}{Z^{\beta+1}(\delta(\sigma(t)))}\left(\frac{r(\sigma(t))}{r(\delta(t))}\right)^{1 / \alpha} \\
& =-\xi(t) \bar{p}(t)+\frac{\xi^{\Delta}(t)}{\xi(\sigma(t))} w(\sigma(t)) \\
& -\frac{\beta \xi(t) \delta^{\Delta}(t)}{(\xi(\sigma(t)))^{1+1 / \alpha}(Z(\delta(\sigma(t))))^{(\alpha-\beta) / \alpha}(r(\delta(t)))^{1 / \alpha}} \\
& \times w^{(\alpha+1) / \alpha}(\sigma(t)) \\
& =-\xi(t) \bar{p}(t)+\frac{\xi^{\Delta}(t)}{\xi(\sigma(t))} w(\sigma(t)) \\
& -\frac{\beta \xi(t) \delta^{\Delta}(t)}{(\xi(\sigma(t)))^{1+1 / \alpha}(Z(\sigma(t)))^{(\alpha-\beta) / \alpha}(r(\delta(t)))^{1 / \alpha}} \\
& \times w^{(\alpha+1) / \alpha}(\sigma(t)) \text {. }
\end{aligned}
$$

Therefore, for $\beta>0$, from (26) and (28), we get

$$
\begin{aligned}
w^{\Delta}(t) \leqslant & -\xi(t) \bar{p}(t)+\frac{\xi^{\Delta}(t)}{\xi(\sigma(t))} w(\sigma(t)) \\
& -\frac{\beta \xi(t) \delta^{\Delta}(t)}{(\xi(\sigma(t)))^{1+1 / \alpha}(Z(\sigma(t)))^{(\alpha-\beta) / \alpha}(r(\delta(t)))^{1 / \alpha}} \\
& \times w^{(\alpha+1) / \alpha}(\sigma(t)) .
\end{aligned}
$$

From (14) and (15), there exists a constant $M_{1}>0$ such that

$$
r(t)\left(Z^{\Delta}(t)\right)^{\alpha} \leqslant M_{1}, \quad t \geqslant T_{1},
$$

that is

$$
Z^{\Delta}(t) \leqslant\left(\frac{M_{1}}{r(t)}\right)^{1 / \alpha}, \quad t \geqslant T_{1},
$$


integrating the above inequality from $T_{1}$ to $t$, we have

$$
Z(t) \leqslant Z\left(T_{1}\right)+M_{1}^{1 / \alpha}\left(R(t)-R\left(T_{1}\right)\right) .
$$

Thus, there exist a constant $M_{2}>0$, and $T_{2} \geqslant T_{1}$ such that

$$
Z(t) \leqslant M_{2} R(t), \quad t \geqslant T_{2}
$$

so we have

$$
\begin{gathered}
Z^{(\alpha-\beta) / \alpha}(\sigma(t)) \leqslant M_{2}^{(\alpha-\beta) / \alpha}(R(\sigma(t)))^{(\alpha-\beta) / \alpha} \\
=M_{3}(R(\sigma(t)))^{(\alpha-\beta) / \alpha}, \quad t \geqslant T_{2}
\end{gathered}
$$

where $M_{3}=M_{2}^{(\alpha-\beta) / \alpha}$.

From (29) and (34), we obtain

$$
\begin{aligned}
w^{\Delta}(t) \leqslant & -\xi(t) \bar{p}(t)+\frac{\xi^{\Delta}(t)}{\xi(\sigma(t))} w(\sigma(t)) \\
& -\frac{\beta \xi(t) \delta^{\Delta}(t)}{(\xi(\sigma(t)))^{1+1 / \alpha} M_{3}(R(\sigma(t)))^{(\alpha-\beta) / \alpha}(r(\delta(t)))^{1 / \alpha}} \\
& \times w^{(\alpha+1) / \alpha}(\sigma(t)), \quad t \geqslant T_{2} .
\end{aligned}
$$

Let

$$
\Psi(t)=\frac{\beta \xi(t) \delta^{\Delta}(t)}{(\xi(\sigma(t)))^{1+1 / \alpha} M_{3}(R(\sigma(t)))^{(\alpha-\beta) / \alpha}(r(\delta(t)))^{1 / \alpha}}
$$

then $\Psi(t)>0$. So from (35) and (36) we get

$$
\begin{aligned}
w^{\Delta}(t) \leqslant & -\xi(t) \bar{p}(t)+\frac{\xi^{\Delta}(t)}{\xi(\sigma(t))} w(\sigma(t)) \\
& -\Psi(t) w^{(\alpha+1) / \alpha}(\sigma(t)) \\
\leqslant & -\xi(t) \bar{p}(t)+\frac{\left(\xi^{\Delta}(t)\right)_{+}}{\xi(\sigma(t))} w(\sigma(t)) \\
& -\Psi(t) w^{(\alpha+1) / \alpha}(\sigma(t)),
\end{aligned}
$$

where $\left(\xi^{\Delta}(t)\right)_{+}:=\max \left\{\xi^{\Delta}(t), 0\right\}$.
Taking $a=\left(\xi^{\Delta}(t)\right)_{+} / \xi(\sigma(t)), b=\Psi(t)$, by Lemma 3 and (37), we obtain

$$
\begin{aligned}
w^{\Delta}(t) & \leqslant-\xi(t) \bar{p}(t)+\frac{\alpha^{\alpha}}{(\alpha+1)^{\alpha+1} \Psi^{\alpha}(t)}\left(\frac{\left(\xi^{\Delta}(t)\right)_{+}}{\xi(\sigma(t))}\right)^{\alpha+1} \\
& =-[\xi(t) \bar{p}(t)
\end{aligned}
$$

$$
\begin{gathered}
\left.-\frac{\alpha^{\alpha}}{(\alpha+1)^{\alpha+1} \Psi^{\alpha}(t)}\left(\frac{\left(\xi^{\Delta}(t)\right)_{+}}{\xi(\sigma(t))}\right)^{\alpha+1}\right] \\
=-[\xi(t) \bar{p}(t) \\
\left.-\frac{\alpha^{\alpha} M_{3}^{\alpha}(R(\sigma(t)))^{\alpha-\beta} r(\delta(t))\left(\left(\xi^{\Delta}(t)\right)_{+}\right)^{\alpha+1}}{(\alpha+1)^{\alpha+1} \beta^{\alpha} \xi^{\alpha}(t)\left(\delta^{\Delta}(t)\right)^{\alpha}}\right] \\
=-\left[\begin{array}{l}
\xi(t) \bar{p}(t)
\end{array}\right]
\end{gathered}
$$

$$
\left.-\frac{\alpha^{\alpha} M_{4}(R(\sigma(t)))^{\alpha-\beta} r(\delta(t))\left(\left(\xi^{\Delta}(t)\right)_{+}\right)^{\alpha+1}}{(\alpha+1)^{\alpha+1} \beta^{\alpha} \xi^{\alpha}(t)\left(\delta^{\Delta}(t)\right)^{\alpha}}\right],
$$

where $M_{4}=M_{3}^{\alpha}$.

Integrating the above inequality (38) from $T_{2}$ to $t$, we have

$$
\begin{gathered}
w(t) \leqslant w\left(T_{2}\right) \\
-\int_{T_{2}}^{t}\left(\xi(s) \bar{p}(s)-\left(\alpha^{\alpha} M_{4}(R(\sigma(s)))^{\alpha-\beta} r(\delta(s))\right.\right. \\
\left.\times\left(\left(\xi^{\Delta}(s)\right)_{+}\right)^{\alpha+1}\right) \\
\left.\times\left((\alpha+1)^{\alpha+1} \beta^{\alpha} \xi^{\alpha}(s)\left(\delta^{\Delta}(s)\right)^{\alpha}\right)^{-1}\right) \Delta s \\
\leqslant w\left(T_{2}\right)+\int_{t_{0}}^{T_{2}} \xi(s) \bar{p}(s) \Delta s
\end{gathered}
$$




$$
\begin{gathered}
-\int_{t_{0}}^{t}\left(\xi(s) \bar{p}(s)-\left(\alpha^{\alpha} M_{4}(R(\sigma(s)))^{\alpha-\beta} r(\delta(s))\right.\right. \\
\left.\times\left(\left(\xi^{\Delta}(s)\right)_{+}\right)^{\alpha+1}\right) \\
\left.\times\left((\alpha+1)^{\alpha+1} \beta^{\alpha} \xi^{\alpha}(s)\left(\delta^{\Delta}(s)\right)^{\alpha}\right)^{-1}\right) \Delta s .
\end{gathered}
$$

Since $w(t)>0$ for $t>T_{2}$, we have

$$
\begin{aligned}
& \int_{t_{0}}^{t}(\xi(s) \bar{p}(s) \\
& \left.\quad-\frac{\alpha^{\alpha} M_{4}(R(\sigma(s)))^{\alpha-\beta} r(\delta(s))\left(\left(\xi^{\Delta}(s)\right)_{+}\right)^{\alpha+1}}{(\alpha+1)^{\alpha+1} \beta^{\alpha} \xi^{\alpha}(s)\left(\delta^{\Delta}(s)\right)^{\alpha}}\right) \Delta s \\
& \leqslant w\left(T_{2}\right)+\int_{t_{0}}^{T_{2}} \xi(s) \bar{p}(s) \Delta s-w(t) \\
& \leqslant w\left(T_{2}\right)+\int_{t_{0}}^{T_{2}} \xi(s) \bar{p}(s) \Delta s
\end{aligned}
$$

which contradicts (12). This completes the proof of Theorem 4 .

Next, we use the general weighted functions from the class $F$ which will be extensively used in the sequel.

Letting $\mathbb{D} \equiv\left\{(t, s) \in \mathbb{T} \times \mathbb{T}: t \geqslant s \geqslant t_{0}\right\}$, we say that a continuous function $H(t, s) \in C_{\mathrm{rd}}(\mathbb{D}, \mathbb{R})$ belongs to the class $F$ if

(i) $H(t, t)=0$ for $t \geqslant t_{0}$ and $H(t, s)>0$ for $t>s \geqslant t_{0}$,

(ii) $H(t, s)$ has a nonpositive right-dense continuous $\Delta$ partial derivative $H^{\Delta_{s}}(t, s)$ with respect to the second variable.

Theorem 5. Assume that $\left(H_{1}\right)-\left(H_{6}\right)$ and $(7)$ hold. If there exist a function $H(t, s) \in F$ and a function $\xi(t) \in C_{\mathrm{rd}}^{1}(\mathbb{T},(0, \infty))$ such that for any positive number $M$,

$$
\varlimsup_{t \rightarrow \infty} \frac{1}{H\left(t, t_{0}\right)} \int_{t_{0}}^{t}[H(t, s) \xi(s) \bar{p}(s)-\widetilde{U}(t, s)] \Delta s=\infty,
$$

where

$$
\begin{aligned}
& \bar{p}(s)=q(s)[1-p(\delta(s))]^{\beta}, \\
& \widetilde{U}(t, s) \\
& =\frac{\alpha^{\alpha}\left(\phi_{+}(t, s)\right)^{\alpha+1}(\xi(\sigma(s)))^{\alpha+1} M(R(\sigma(s)))^{\alpha-\beta} r(\delta(s))}{(\alpha+1)^{\alpha+1} \beta^{\alpha}(H(t, s))^{\alpha} \xi^{\alpha}(s)\left(\delta^{\Delta}(s)\right)^{\alpha}}
\end{aligned}
$$

$$
\begin{aligned}
& \phi_{+}(t, s):=\max \left\{H^{\Delta_{s}}(t, s)+\frac{H(t, s)\left(\xi^{\Delta}(s)\right)_{+}}{\xi(\sigma(s))}, 0\right\}, \\
& \left(\xi^{\Delta}(s)\right)_{+}:=\max \left\{\xi^{\Delta}(s), 0\right\},
\end{aligned}
$$

then (1) is oscillatory.

Proof. We proceed as in the proof of Theorem 4 to have (37). From (37) we obtain

$$
\begin{aligned}
\xi(t) \bar{p}(t) \leqslant & -w^{\Delta}(t)+\frac{\left(\xi^{\Delta}(t)\right)_{+}}{\xi(\sigma(t))} w(\sigma(t)) \\
& -\Psi(t) w^{(\alpha+1) / \alpha}(\sigma(t)), \quad t \geqslant T_{2} .
\end{aligned}
$$

Multiplying (46) (with $t$ replaced by $s$ ) by $H(t, s)$, integrating it with respect to $s$ from $T_{2}$ to $t$ for $t>T_{2}$, using integration by parts and (i)-(ii), we get

$$
\begin{aligned}
& \int_{T_{2}}^{t} H(t, s) \xi(s) \bar{p}(s) \Delta s \\
& \leqslant-\int_{T_{2}}^{t} H(t, s) w^{\Delta}(s) \Delta s \\
& +\int_{T_{2}}^{t} \frac{H(t, s)\left(\xi^{\Delta}(s)\right)_{+}}{\xi(\sigma(s))} w(\sigma(s)) \Delta s \\
& -\int_{T_{2}}^{t} H(t, s) \Psi(s) w^{(\alpha+1) / \alpha}(\sigma(s)) \Delta s \\
& =H\left(t, T_{2}\right) w\left(T_{2}\right)+\int_{T_{2}}^{t} H^{\Delta_{s}}(t, s) w(\sigma(s)) \Delta s \\
& +\int_{T_{2}}^{t} \frac{H(t, s)\left(\xi^{\Delta}(s)\right)_{+}}{\xi(\sigma(s))} w(\sigma(s)) \Delta s \\
& -\int_{T_{2}}^{t} H(t, s) \Psi(s) w^{(\alpha+1) / \alpha}(\sigma(s)) \Delta s \\
& =H\left(t, T_{2}\right) w\left(T_{2}\right) \\
& +\int_{T_{2}}^{t}\left(H^{\Delta_{s}}(t, s)+\frac{H(t, s)\left(\xi^{\Delta}(s)\right)_{+}}{\xi(\sigma(s))}\right) w(\sigma(s)) \Delta s \\
& -\int_{T_{2}}^{t} H(t, s) \Psi(s) w^{(\alpha+1) / \alpha}(\sigma(s)) \Delta s \\
& =H\left(t, T_{2}\right) w\left(T_{2}\right) \\
& +\int_{T_{2}}^{t}\left[\left(H^{\Delta_{s}}(t, s)+\frac{H(t, s)\left(\xi^{\Delta}(s)\right)_{+}}{\xi(\sigma(s))}\right) w(\sigma(s))\right.
\end{aligned}
$$




$$
\begin{gathered}
\left.-H(t, s) \Psi(s) w^{(\alpha+1) / \alpha}(\sigma(s))\right] \Delta s \\
\leqslant H\left(t, T_{2}\right) w\left(T_{2}\right) \\
+\int_{T_{2}}^{t}\left[\begin{array}{l}
\phi_{+}(t, s) w(\sigma(s)) \\
\left.-H(t, s) \Psi(s) w^{(\alpha+1) / \alpha}(\sigma(s))\right] \Delta s,
\end{array}\right.
\end{gathered}
$$

where $\phi_{+}(t, s)$ is defined as in (44).

Taking $a=\phi_{+}(t, s), b=H(t, s) \Psi(s)$, by Lemma 3 and (47), we obtain

$$
\begin{aligned}
& \int_{T_{2}}^{t} H(t, s) \xi(s) \bar{p}(s) \Delta s \\
& \leqslant H\left(t, T_{2}\right) w\left(T_{2}\right) \\
& +\int_{T_{2}}^{t}\left[\left(\alpha^{\alpha}\left(\phi_{+}(t, s)\right)^{\alpha+1}(\xi(\sigma(s)))^{\alpha+1}\right.\right. \\
& \left.\times M_{3}^{\alpha}(R(\sigma(s)))^{\alpha-\beta} r(\delta(s))\right) \\
& \times\left((\alpha+1)^{\alpha+1} \beta^{\alpha}(H(t, s))^{\alpha}\right. \\
& \left.\left.\times \xi^{\alpha}(s)\left(\delta^{\Delta}(s)\right)^{\alpha}\right)^{-1}\right] \Delta s \\
& \leqslant H\left(t, T_{2}\right) w\left(T_{2}\right) \\
& +\int_{T_{2}}^{t}\left[\left(\alpha^{\alpha}\left(\phi_{+}(t, s)\right)^{\alpha+1}(\xi(\sigma(s)))^{\alpha+1}\right.\right. \\
& \left.\times M_{4}(R(\sigma(s)))^{\alpha-\beta} r(\delta(s))\right) \\
& \times\left((\alpha+1)^{\alpha+1} \beta^{\alpha}(H(t, s))^{\alpha}\right. \\
& \left.\left.\times \xi^{\alpha}(s)\left(\delta^{\Delta}(s)\right)^{\alpha}\right)^{-1}\right] \Delta s \\
& \leqslant H\left(t, t_{0}\right) w\left(T_{2}\right)+\int_{T_{2}}^{t} U(t, s) \Delta s,
\end{aligned}
$$

where $M_{4}=M_{3}^{\alpha}$,

$$
\begin{aligned}
& U(t, s) \\
& \quad=\frac{\alpha^{\alpha}\left(\phi_{+}(t, s)\right)^{\alpha+1}(\xi(\sigma(s)))^{\alpha+1} M_{4}(R(\sigma(s)))^{\alpha-\beta} r(\delta(s))}{(\alpha+1)^{\alpha+1} \beta^{\alpha}(H(t, s))^{\alpha} \xi^{\alpha}(s)\left(\delta^{\Delta}(s)\right)^{\alpha}} .
\end{aligned}
$$

Then it follows that

$$
\frac{1}{H\left(t, t_{0}\right)} \int_{T_{2}}^{t}[H(t, s) \xi(s) \bar{p}(s)-U(t, s)] \Delta s \leqslant w\left(T_{2}\right) .
$$

Thus we get

$$
\begin{aligned}
& \frac{1}{H\left(t, t_{0}\right)} \int_{t_{0}}^{t}[H(t, s) \xi(s) \bar{p}(s)-U(t, s)] \Delta s \\
& \quad=\frac{1}{H\left(t, t_{0}\right)}\left(\int_{t_{0}}^{T_{2}}+\int_{T_{2}}^{t}\right)[H(t, s) \xi(s) \bar{p}(s)-U(t, s)] \Delta s \\
& \quad \leqslant w\left(T_{2}\right)+\frac{1}{H\left(t, t_{0}\right)} \int_{t_{0}}^{T_{2}}[H(t, s) \xi(s) \bar{p}(s)-U(t, s)] \Delta s \\
& \quad \leqslant w\left(T_{2}\right)+\int_{t_{0}}^{T_{2}}\left[\frac{H(t, s)}{H\left(t, t_{0}\right)} \xi(s) \bar{p}(s)-\frac{U(t, s)}{H\left(t, t_{0}\right)}\right] \Delta s \\
& \quad \leqslant w\left(T_{2}\right)+\int_{t_{0}}^{T_{2}} \xi(s) \bar{p}(s) \Delta s .
\end{aligned}
$$

Then

$$
\varlimsup_{t \rightarrow \infty} \frac{1}{H\left(t, t_{0}\right)} \int_{t_{0}}^{t}[H(t, s) \xi(s) \bar{p}(s)-U(t, s)] \Delta s<\infty,
$$

which contradicts (41). This completes the proof of Theorem 5 .

Theorem 6. Assume that $\left(H_{1}\right)-\left(H_{6}\right)$ and $(7)$ hold and $\beta \geqslant 1$. Furthermore, assume that $r^{\Delta}(t) \geqslant 0$. If there exists a function $\xi(t) \in C_{\mathrm{rd}}^{1}(\mathbb{T},(0, \infty))$ such that for any positive number $M$,

$$
\varlimsup_{t \rightarrow \infty} \int_{t_{0}}^{t}(\xi(s) \bar{p}(s)-Q(s)) \Delta s=\infty,
$$

where

$$
\begin{gathered}
\bar{p}(s)=q(s)[1-p(\delta(s))]^{\beta}, \\
Q(s)=\frac{\left(\xi^{\Delta}(s)\right)^{2}(r(\sigma(s)))^{(\alpha-\beta) / \alpha}(r(\delta(s)))^{\beta / \alpha}}{4 \beta \xi(s)(\delta(s) / 2)^{\beta-1} \delta^{\Delta}(s) M^{\alpha-\beta}},
\end{gathered}
$$

then (1) is oscillatory.

Proof. We proceed as in the proof of Theorem 4 to have (24). On the other hand, from $(22)$ and $\left(\mathrm{H}_{3}\right)$, we deduce

$$
\left(r(t)\left(Z^{\Delta}(t)\right)^{\alpha}\right)^{\Delta} \leqslant 0, \quad t \geqslant T_{1},
$$

and from $r^{\Delta}(t) \geqslant 0$ for $t \geqslant t_{0}$, we can get $Z^{\Delta}(t)$ is nonincreasing. Hence, we have

$$
Z(t)-Z\left(T_{1}\right)=\int_{T_{1}}^{t} Z^{\Delta}(s) \Delta s \geqslant\left(t-T_{1}\right) Z^{\Delta}(t),
$$


which implies

$$
Z(t) \geqslant \frac{t}{2} Z^{\Delta}(t), \quad \text { for } t \geqslant T_{2}>2 T_{1} .
$$

Choosing $T_{3} \geqslant T_{2}$ such that $\delta(t) \geqslant T_{2}$ for $t \geqslant T_{3}$, we get

$$
Z(\delta(t)) \geqslant \frac{\delta(t)}{2} Z^{\Delta}(\delta(t)), \quad \text { for } t \geqslant T_{3} .
$$

From $\left(\mathrm{H}_{6}\right),(15),(20),(24),(25),(58)$, and as $Z^{\Delta}(t)$ is nonincreasing, we obtain

$$
\begin{aligned}
w^{\Delta}(t) \leqslant & -\xi(t) \bar{p}(t)+\frac{\xi^{\Delta}(t)}{\xi(\sigma(t))} w(\sigma(t)) \\
& -\left(r(\sigma(t))\left(Z^{\Delta}(\sigma(t))\right)^{\alpha} \xi(t) \beta(Z(\delta(t)))^{\beta-1}\right. \\
& \left.\times Z^{\Delta}(\delta(t)) \delta^{\Delta}(t)\right)\left(Z^{2 \beta}(\delta(\sigma(t)))\right)^{-1} \\
\leqslant & -\xi(t) \bar{p}(t)+\frac{\xi^{\Delta}(t)}{\xi(\sigma(t))} w(\sigma(t)) \\
& -\left(r(\sigma(t))\left(Z^{\Delta}(\sigma(t))\right)^{\alpha} \xi(t)\right. \\
& \times\left(Z^{2 \beta}(\delta(\sigma(t)))\right)^{-1} \\
\leqslant & -\xi(t) \bar{p}(t)+\frac{\xi^{\Delta}(t)}{\xi(\sigma(t))} w(\sigma(t)) \\
& -\left(\beta \xi(t) r(\sigma(t))\left(Z^{\Delta}(\sigma(t))\right)^{\alpha+\beta}(\delta(t) / 2)^{\beta-1} \delta^{\Delta}(t)\right) \\
& \times\left(\xi^{2}(\sigma(t))(r(\sigma(t)))^{(\alpha-\beta) / \alpha}\left(Z^{\Delta}(\sigma(t))\right)^{\alpha-\beta}\right. \\
& \left.\times\left(Z^{2 \beta}(\delta(\sigma(t)))\right)^{-1}\left(\frac{r(\sigma(t))}{r(\delta(t))}\right)^{\beta / \alpha}(\delta(t)) \delta^{\Delta}(t)\right) \\
= & -\xi(t) \bar{p}(t)+\frac{\xi^{\Delta}(t)}{\xi(\sigma(t))} w(\sigma(t)) \\
& -\left(\beta \xi(t)(\delta(t) / 2)^{\beta-1} \delta^{\Delta}(t)\right) \\
& \\
& \\
& \\
& \\
& \\
&
\end{aligned}
$$

Now, from the fact that $Z^{\Delta}(t)$ is nonnegative and nonincreasing, there exists a $T_{4}>T_{3}$ sufficiently large such that

$$
Z^{\Delta}(t) \leqslant \frac{1}{M}, \quad t \geqslant T_{4},
$$

holds for some positive constant $M$ and therefore

$$
\left(Z^{\Delta}(t)\right)^{\alpha-\beta} \leqslant\left(\frac{1}{M}\right)^{\alpha-\beta}, \quad t \geqslant T_{4} .
$$

Combining (59) and (61), we obtain that

$$
\begin{aligned}
w^{\Delta}(t) \leqslant & -\xi(t) \bar{p}(t)+\frac{\xi^{\Delta}(t)}{\xi(\sigma(t))} w(\sigma(t)) \\
& -\frac{\beta \xi(t)(\delta(t) / 2)^{\beta-1} \delta^{\Delta}(t) M^{\alpha-\beta}}{\xi^{2}(\sigma(t))(r(\sigma(t)))^{(\alpha-\beta) / \alpha}(r(\delta(t)))^{\beta / \alpha}} \\
& \times w^{2}(\sigma(t)), \quad t \geqslant T_{4} .
\end{aligned}
$$

Letting

$$
\Phi(t)=\frac{\beta \xi(t)(\delta(t) / 2)^{\beta-1} \delta^{\Delta}(t) M^{\alpha-\beta}}{\xi^{2}(\sigma(t))(r(\sigma(t)))^{(\alpha-\beta) / \alpha}(r(\delta(t)))^{\beta / \alpha}},
$$

then $\Phi(t) \geqslant 0$. So

$$
\begin{aligned}
& w^{\Delta}(t) \leqslant-\xi(t) \bar{p}(t)+\frac{\xi^{\Delta}(t)}{\xi(\sigma(t))} w(\sigma(t))-\Phi(t) w^{2}(\sigma(t)) \\
&=-\xi(t) \bar{p}(t)+\frac{1}{4 \Phi(t)} \frac{\left(\xi^{\Delta}(t)\right)^{2}}{\xi^{2}(\sigma(t))} \\
&-\left[\sqrt{\Phi(t)} w(\sigma(t))-\frac{1}{2 \sqrt{\Phi(t)}} \frac{\xi^{\Delta}(t)}{\xi(\sigma(t))}\right]^{2} \\
& \leqslant-\xi(t) \bar{p}(t)+\frac{1}{4 \Phi(t)} \frac{\left(\xi^{\Delta}(t)\right)^{2}}{\xi^{2}(\sigma(t))} \\
&=-[\xi(t) \bar{p}(t) \\
&\left.-\frac{\left(\xi^{\Delta}(t)\right)^{2}(r(\sigma(t)))^{(\alpha-\beta) / \alpha}(r(\delta(t)))^{\beta / \alpha}}{4 \beta \xi(t)(\delta(t) / 2)^{\beta-1} \delta^{\Delta}(t) M^{\alpha-\beta}}\right] .
\end{aligned}
$$


Integrating the above inequality from $T_{4}$ to $t$, we have

$w(t) \leqslant w\left(T_{4}\right)$

$$
\begin{aligned}
&-\int_{T_{4}}^{t}(\xi(s) \bar{p}(s) \\
&-\left(\left(\xi^{\Delta}(s)\right)^{2}(r(\sigma(s)))^{(\alpha-\beta) / \alpha}(r(\delta(s)))^{\beta / \alpha}\right) \\
&\left.\times\left(4 \beta \xi(s)(\delta(s) / 2)^{\beta-1} \delta^{\Delta}(s) M^{\alpha-\beta}\right)^{-1}\right) \Delta s \\
& \leqslant\left(T_{4}\right)+ \int_{t_{0}}^{T_{4}} \xi(s) \bar{p}(s) \Delta s \\
&-\int_{t_{0}}^{t}\left(\begin{array}{l}
\xi \\
(s) \bar{p}(s) \\
-\left(\left(\xi^{\Delta}(s)\right)^{2}(r(\sigma(s)))^{(\alpha-\beta) / \alpha}(r(\delta(s)))^{\beta / \alpha}\right) \\
\left.\times\left(4 \beta \xi(s)(\delta(s) / 2)^{\beta-1} \delta^{\Delta}(s) M^{\alpha-\beta}\right)^{-1}\right) \Delta s .
\end{array}\right.
\end{aligned}
$$

Since $w(t)>0$ for $t>T_{4}$, we have

$$
\begin{gathered}
\int_{t_{0}}^{t}\left(\xi(s) \bar{p}(s)-\frac{\left(\xi^{\Delta}(s)\right)^{2}(r(\sigma(s)))^{(\alpha-\beta) / \alpha}(r(\delta(s)))^{\beta / \alpha}}{4 \beta \xi(s)(\delta(s) / 2)^{\beta-1} \delta^{\Delta}(s) M^{\alpha-\beta}}\right) \Delta s \\
\leqslant w\left(T_{4}\right)+\int_{t_{0}}^{T_{4}} \xi(s) \bar{p}(s) \Delta s-w(t) \\
<w\left(T_{4}\right)+\int_{t_{0}}^{T_{4}} \xi(s) \bar{p}(s) \Delta s .
\end{gathered}
$$

which contradicts (53). This completes the proof of Theorem 6.

Theorem 7. Assume that $\left(H_{1}\right)-\left(H_{6}\right)$ and (7) hold and $\beta \geqslant 1$. Furthermore, assume that $r^{\Delta}(t) \geqslant 0$. If there exist a function $H(t, s) \in F$ and a function $\xi(t) \in C_{\mathrm{rd}}^{1}(\mathbb{T},(0, \infty))$ such that

$$
\begin{array}{r}
H^{\Delta_{s}}(t, s)+\frac{H(t, s) \xi^{\Delta}(s)}{\xi(\sigma(s))} \leqslant 0, \text { for } t \geqslant s \geqslant t_{0}, \\
\varlimsup_{t \rightarrow \infty} \frac{1}{H\left(t, t_{0}\right)} \int_{t_{0}}^{t} H(t, s) \xi(s) \bar{p}(s) \Delta s=\infty,
\end{array}
$$

where

$$
\bar{p}(s)=q(s)[1-p(\delta(s))]^{\beta},
$$

then (1) is oscillatory.
Proof. We proceed as in the proof of Theorem 6 to have (64). From (64) we obtain

$$
\begin{aligned}
\xi(t) \bar{p}(t) \leqslant & -w^{\Delta}(t)+\frac{\xi^{\Delta}(t)}{\xi(\sigma(t))} w(\sigma(t)) \\
& -\Phi(t) w^{2}(\sigma(t)), \quad t \geqslant T_{4} .
\end{aligned}
$$

Multiplying (70) (with $t$ replaced by $s$ ) by $H(t, s)$, integrating it with respect to $s$ from $T_{4}$ to $t$ for $t>T_{4}$, using integration by parts and (i)-(ii), we get

$$
\begin{aligned}
\int_{T_{4}}^{t} H(t, s) \xi(s) \bar{p}(s) \Delta s \\
\leqslant-\int_{T_{4}}^{t} H(t, s) w^{\Delta}(s) \Delta s+\int_{T_{4}}^{t} \frac{H(t, s) \xi^{\Delta}(s)}{\xi(\sigma(s))} w(\sigma(s)) \Delta s \\
\quad-\int_{T_{4}}^{t} H(t, s) \Phi(s) w^{2}(\sigma(s)) \Delta s \\
=\quad H\left(t, T_{4}\right) w\left(T_{4}\right)+\int_{T_{4}}^{t} H^{\Delta_{s}}(t, s) w(\sigma(s)) \Delta s \\
\quad+\int_{T_{4}}^{t} \frac{H(t, s) \xi^{\Delta}(s)}{\xi(\sigma(s))} w^{\prime}(\sigma(s)) \Delta s \\
\quad-\int_{T_{4}}^{t} H(t, s) \Phi(s) w^{2}(\sigma(s)) \Delta s \\
=\quad H\left(t, T_{4}\right) w\left(T_{4}\right) \\
\quad+\int_{T_{4}}^{t}\left(H^{\Delta_{s}}(t, s)+\frac{H(t, s) \xi^{\Delta}(s)}{\xi(\sigma(s))}\right) w(\sigma(s)) \Delta s \\
\quad-\int_{T_{4}}^{t} H(t, s) \Phi(s) w^{2}(\sigma(s)) \Delta s .
\end{aligned}
$$

Using (67) in the above inequality (71), we get

$$
\int_{T_{4}}^{t} H(t, s) \xi(s) \bar{p}(s) \Delta s \leqslant H\left(t, t_{0}\right) w\left(T_{4}\right) .
$$

Then it follows that

$$
\frac{1}{H\left(t, t_{0}\right)} \int_{T_{4}}^{t} H(t, s) \xi(s) \bar{p}(s) \Delta s \leqslant w\left(T_{4}\right) .
$$


Thus we get

$$
\begin{aligned}
& \frac{1}{H\left(t, t_{0}\right)} \int_{t_{0}}^{t} H(t, s) \xi(s) \bar{p}(s) \Delta s \\
& \quad=\frac{1}{H\left(t, t_{0}\right)}\left(\int_{t_{0}}^{T_{4}}+\int_{T_{4}}^{t}\right) H(t, s) \xi(s) \bar{p}(s) \Delta s \\
& \quad \leqslant w\left(T_{4}\right)+\frac{1}{H\left(t, t_{0}\right)} \int_{t_{0}}^{T_{4}} H(t, s) \xi(s) \bar{p}(s) \Delta s \\
& \quad \leqslant w\left(T_{4}\right)+\int_{t_{0}}^{T_{4}} \frac{H(t, s)}{H\left(t, t_{0}\right)} \xi(s) \bar{p}(s) \Delta s \\
& \quad \leqslant w\left(T_{4}\right)+\int_{t_{0}}^{T_{4}} \xi(s) \bar{p}(s) \Delta s .
\end{aligned}
$$

Then

$$
\varlimsup_{t \rightarrow \infty} \frac{1}{H\left(t, t_{0}\right)} \int_{t_{0}}^{t} H(t, s) \xi(s) \bar{p}(s) \Delta s<\infty
$$

which contradicts (68). This completes the proof of Theorem 7 .

Theorem 8. Assume that $\left(H_{1}\right)-\left(H_{6}\right)$ and (7) hold and $\beta \geqslant 1$. Furthermore, assume that $r^{\Delta}(t) \geqslant 0$. If there exist a function $H(t, s) \in F$ and a function $\xi(t) \in C_{\mathrm{rd}}^{1}(\mathbb{T},(0, \infty))$ such that for any positive number $M$,

$$
\begin{aligned}
\varlimsup_{t \rightarrow \infty} & \frac{1}{H\left(t, t_{0}\right)} \\
\times \int_{t_{0}}^{t}[ & H(t, s) \xi(s) \bar{p}(s) \\
& \left.-\frac{\left(H^{\Delta_{s}}(t, s)+H(t, s) \xi^{\Delta}(s) / \xi(\sigma(s))\right)^{2}}{4 H(t, s) \Phi(s)}\right] \Delta s=\infty,
\end{aligned}
$$

where

$$
\begin{gathered}
\bar{p}(s)=q(s)[1-p(\delta(s))]^{\beta}, \\
\Phi(s)=\frac{\beta \xi(s)(\delta(s) / 2)^{\beta-1} \delta^{\Delta}(s) M^{\alpha-\beta}}{\xi^{2}(\sigma(s))(r(\sigma(s)))^{(\alpha-\beta) / \alpha}(r(\delta(s)))^{\beta / \alpha}}
\end{gathered}
$$

then (1) is oscillatory.
Proof. We proceed as those in the proof of Theorem 7 to have (71), that is,

$$
\begin{aligned}
& \int_{T_{4}}^{t} H(t, s) \xi(s) \bar{p}(s) \Delta s \\
& \leqslant H\left(t, T_{4}\right) w\left(T_{4}\right) \\
& +\int_{T_{4}}^{t}\left(H^{\Delta_{s}}(t, s)+\frac{H(t, s) \xi^{\Delta}(s)}{\xi(\sigma(s))}\right) w(\sigma(s)) \Delta s \\
& -\int_{T_{4}}^{t} H(t, s) \Phi(s) w^{2}(\sigma(s)) \Delta s \\
& =H\left(t, T_{4}\right) w\left(T_{4}\right) \\
& +\int_{T_{4}}^{t} \frac{\left(H^{\Delta_{s}}(t, s)+H(t, s) \xi^{\Delta}(s) / \xi(\sigma(s))\right)^{2}}{4 H(t, s) \Phi(s)} \Delta s \\
& -\int_{T_{4}}^{t}\left[\frac{H^{\Delta_{s}}(t, s)+H(t, s) \xi^{\Delta}(s) / \xi(\sigma(s))}{2 \sqrt{H(t, s) \Phi(s)}}\right. \\
& -\sqrt{H(t, s) \Phi(s)} w(\sigma(s))]^{2} \Delta s \\
& \leqslant H\left(t, T_{4}\right) w\left(T_{4}\right) \\
& +\int_{T_{4}}^{t} \frac{\left(H^{\Delta_{s}}(t, s)+H(t, s) \xi^{\Delta}(s) / \xi(\sigma(s))\right)^{2}}{4 H(t, s) \Phi(s)} \Delta s \\
& \leqslant H\left(t, t_{0}\right) w\left(T_{4}\right) \\
& +\int_{T_{4}}^{t} \frac{\left(H^{\Delta_{s}}(t, s)+H(t, s) \xi^{\Delta}(s) / \xi(\sigma(s))\right)^{2}}{4 H(t, s) \Phi(s)} \Delta s .
\end{aligned}
$$

Then it follows that

$$
\begin{aligned}
& \frac{1}{H\left(t, t_{0}\right)} \\
& \times \int_{T_{4}}^{t}[H(t, s) \xi(s) \bar{p}(s) \\
& \leqslant w\left(T_{4}\right) . \\
& \frac{1}{H\left(t, t_{0}\right)} \int_{t_{0}}^{t}[H(t, s) \xi(s) \bar{p}(s) \\
& -\left(H^{\Delta_{s}}(t, s)+\frac{H(t, s) \xi^{\Delta}(s)}{\xi(\sigma(s))}\right)^{2}
\end{aligned}
$$$$
\left.-\frac{\left(H^{\Delta_{s}}(t, s)+H(t, s) \xi^{\Delta}(s) / \xi(\sigma(s))\right)^{2}}{4 H(t, s) \Phi(s)}\right] \Delta s
$$ 


$$
\begin{aligned}
& \left.\times(4 H(t, s) \Phi(s))^{-1}\right] \Delta s \\
& =\frac{1}{H\left(t, t_{0}\right)} \\
& \times\left\{\int_{t_{0}}^{T_{4}}+\int_{T_{4}}^{t}\right\}[H(t, s) \xi(s) \bar{p}(s) \\
& -\left(H^{\Delta_{s}}(t, s)+\frac{H(t, s) \xi^{\Delta}(s)}{\xi(\sigma(s))}\right)^{2} \\
& \left.\times(4 H(t, s) \Phi(s))^{-1}\right] \Delta s \\
& \leqslant w\left(T_{4}\right)+\frac{1}{H\left(t, t_{0}\right)} \\
& \times \int_{t_{0}}^{T_{4}}[H(t, s) \xi(s) \bar{p}(s) \\
& -\left(H^{\Delta_{s}}(t, s)+\frac{H(t, s) \xi^{\Delta}(s)}{\xi(\sigma(s))}\right)^{2} \\
& \left.\times(4 H(t, s) \Phi(s))^{-1}\right] \Delta s
\end{aligned}
$$$$
\leqslant w\left(T_{4}\right)
$$$$
+\int_{t_{0}}^{T_{4}}\left[\frac{H(t, s)}{H\left(t, t_{0}\right)} \xi(s) \bar{p}(s)\right.
$$$$
-\left(H^{\Delta_{s}}(t, s)+\frac{H(t, s) \xi^{\Delta}(s)}{\xi(\sigma(s))}\right)^{2}
$$$$
\left.\times\left(4 H(t, s) H\left(t, t_{0}\right) \Phi(s)\right)^{-1}\right] \Delta s
$$$$
\leqslant w\left(T_{4}\right)+\int_{t_{0}}^{T_{4}} \xi(s) \bar{p}(s) \Delta s .
$$

Then

$$
\begin{aligned}
& \varlimsup_{t \rightarrow \infty} \frac{1}{H\left(t, t_{0}\right)} \\
& \times \int_{t_{0}}^{t}[H(t, s) \xi(s) \bar{p}(s) \\
&\left.\quad-\frac{\left(H^{\Delta_{s}}(t, s)+H(t, s) \xi^{\Delta}(s) / \xi(\sigma(s))\right)^{2}}{4 H(t, s) \Phi(s)}\right] \Delta s
\end{aligned}
$$$$
<\infty
$$

which contradicts (76). This completes the proof of Theorem 8 .

The case

$$
\lim _{t \rightarrow \infty} \int_{t_{0}}^{t} r^{-1 / \alpha}(s) \Delta s<\infty .
$$

Theorem 9. Assume that $\left(H_{1}\right)-\left(H_{6}\right)$ and (8) hold and there exists a $T_{*} \in\left[t_{0}, \infty\right)_{\mathbb{T}}$ such that $p^{\Delta}(t) \geqslant 0, \tau^{\Delta}(t) \geqslant 0$ for $t \geqslant T_{*}$, and suppose that there exists a function $\xi(t) \in$ $C_{\mathrm{rd}}^{1}(\mathbb{T},(0, \infty))$ such that $(12)$ holds for any positive number $M$, and there exists a function $\psi(t) \in C_{\mathrm{rd}}^{1}(\mathbb{T},(0, \infty))$ satisfying $\psi(t) \geqslant t, \psi^{\Delta}(t)>0, \delta(t) \leqslant \tau(\psi(t))$ for $t \geqslant T_{*}$ such that for any positive number $M$ and for every $T_{1} \in\left[T_{*}, \infty\right)_{\mathbb{T}}$

$$
\varlimsup_{t \rightarrow \infty} \int_{T_{1}}^{t}\left[\tilde{p}(s) V^{\alpha}(\sigma(s))-G(s)\right] \Delta s=\infty,
$$

where

$$
\begin{aligned}
& \widetilde{p}(s)=q(s)\left(\frac{1}{1+p(\psi(s))}\right)^{\beta}, \\
& V(s)=\int_{\psi(s)}^{\infty} r^{-1 / \alpha}(t) \Delta t, \\
& G(s)
\end{aligned}
$$

$$
= \begin{cases}\frac{\alpha^{2 \alpha+1} r^{-1 / \alpha}(\psi(s)) \psi^{\Delta}(s)}{(\alpha+1)^{\alpha+1} \beta^{\alpha} M^{\alpha-\beta} V(\sigma(s))}, & \text { if } 0<\alpha<1, \\ \frac{\alpha^{2 \alpha+1} r^{-1 / \alpha}(\psi(s)) V^{\alpha^{2}-1}(s) \psi^{\Delta}(s)}{(\alpha+1)^{\alpha+1} \beta^{\alpha} M^{\alpha-\beta} V^{\alpha^{2}}(\sigma(s))}, & \text { if } \alpha \geqslant 1,\end{cases}
$$

then (1) is oscillatory.

Proof. Suppose to the contrary that $x(t)$ is an eventually positive solution of (1), then there exists a $T_{1} \geqslant T_{*} \geqslant t_{0}$ such that $x(t)>0, x(\delta(t))>0, x(\sigma(t))>0$ for all $t \geqslant T_{1}$, (the case of $x(t)$ is negative and can be considered by the same method). It follows form $\left(\mathrm{H}_{3}\right)$ that $Z(t) \geqslant x(t)>0$ for $t \geqslant T_{1}$. From (14) it is easy to conclude that there exist two possible cases of the sign of $Z^{\Delta}(t)$.

Case 1. Suppose $Z^{\Delta}(t) \geqslant 0$ for sufficiently large $t$, then we are back to the case of Theorem 4 . Thus the proof of Theorem 4 goes through, and we may get contradiction by (12).

Case 2. Suppose $Z^{\Delta}(t)<0$ for $t \geqslant T_{1}$. Define

$$
w(t)=\frac{r(t)\left(-Z^{\Delta}(t)\right)^{\alpha-1} Z^{\Delta}(t)}{Z^{\beta}(\psi(t))}, \quad t \geqslant T_{1} .
$$

Then $w(t)<0$ for $t \geqslant T_{1}$. From the fact that $Z(t)$ is positive and nonincreasing, we get that

$$
Z(\psi(t)) \leqslant \frac{1}{M_{0}}, \quad t \geqslant T_{1},
$$


holds for some positive constant $M_{0}$.

Noting that $\left(r(t)\left(-Z^{\Delta}(t)\right)^{\alpha-1} Z^{\Delta}(t)\right)^{\Delta} \leqslant 0, \psi(t) \geqslant t$, so we have

$$
\begin{gathered}
Z^{\Delta}(\psi(t)) \leqslant\left(\frac{r(t)}{r(\psi(t))}\right)^{1 / \alpha} Z^{\Delta}(t), \\
Z^{\Delta}(s) \leqslant \frac{r^{1 / \alpha}(t)}{r^{1 / \alpha}(s)} Z^{\Delta}(t), \quad s \geqslant t .
\end{gathered}
$$

Integrating the above inequality (88) with respect to $s$ from $\psi(t)$ to $v$, we have

$$
Z(v) \leqslant Z(\psi(t))+r^{1 / \alpha}(t) Z^{\Delta}(t) \int_{\psi(t)}^{v} r^{1 / \alpha}(s) \Delta s
$$

Letting $v \rightarrow \infty$ in the above inequality, we obtain

$$
0 \leqslant Z(\psi(t))+r^{1 / \alpha}(t) Z^{\Delta}(t) V(t) .
$$

From (86) and (90), we have

$$
-\frac{1}{M_{0}^{\alpha-\beta}} \leqslant w(t) V^{\alpha}(t) \leqslant 0, \quad t \geqslant T_{1} .
$$

If $0<\beta<1$. From $Z^{\Delta}(t)<0$, Lemmas 1 and 2 , we have

$$
\begin{aligned}
\left(Z^{\beta}(\psi(t))\right)^{\Delta} \\
=\beta\left\{\int_{0}^{1}[(1-h) Z(\psi(t))+h Z(\psi(\sigma(t)))]^{\beta-1} \mathrm{~d} h\right\} \\
\quad \times(Z(\psi(t)))^{\Delta} \\
\quad \leqslant \beta\left[\int_{0}^{1} Z^{\beta-1}(\psi(t)) \mathrm{d} h\right] Z^{\Delta}(\psi(t)) \psi^{\Delta}(t) \\
=\beta Z^{\beta-1}(\psi(t)) Z^{\Delta}(\psi(t)) \psi^{\Delta}(t) .
\end{aligned}
$$

From (1), $\left(\mathrm{H}_{6}\right),(85)$, and (92), we get

$$
\begin{aligned}
& w^{\Delta}(t) \\
& =\frac{1}{Z^{\beta}(\psi(t))}\left(r(t)\left(-Z^{\Delta}(t)\right)^{\alpha-1} Z^{\Delta}(t)\right)^{\Delta} \\
& \quad-\left(r(\sigma(t))\left(-Z^{\Delta}(\sigma(t))\right)^{\alpha-1} Z^{\Delta}(\sigma(t))\left(Z^{\beta}(\psi(t))\right)^{\Delta}\right)
\end{aligned}
$$

$$
\begin{aligned}
& \times\left(Z^{\beta}(\psi(t)) Z^{\beta}(\psi(\sigma(t)))\right)^{-1} \\
\leqslant & -q(t) \frac{x^{\beta}(\delta(t))}{Z^{\beta}(\psi(t))} \\
& -\left(r(\sigma(t))\left(-Z^{\Delta}(\sigma(t))\right)^{\alpha-1} Z^{\Delta}(\sigma(t)) \beta Z^{\beta-1}\right. \\
& \left.\times(\psi(t)) Z^{\Delta}(\psi(t)) \psi^{\Delta}(t)\right) \\
\times & \left(Z^{\beta}(\psi(t)) Z^{\beta}(\psi(\sigma(t)))\right)^{-1} \\
\leqslant & -q(t) \frac{x^{\beta}(\delta(t))}{Z^{\beta}(\psi(t))} \\
- & \left(r(\sigma(t))\left(-Z^{\Delta}(\sigma(t))\right)^{\alpha-1} Z^{\Delta}(\sigma(t))\right. \\
& \left.\times \beta Z^{\Delta}(\psi(t)) \psi^{\Delta}(t)\right)\left(Z(\psi(t)) Z^{\beta}(\psi(\sigma(t)))\right)^{-1}
\end{aligned}
$$$$
\leqslant-q(t) \frac{x^{\beta}(\delta(t))}{Z^{\beta}(\psi(t))}
$$$$
-\left(r(\sigma(t))\left(-Z^{\Delta}(\sigma(t))\right)^{\alpha-1} Z^{\Delta}(\sigma(t))\right.
$$$$
\left.\times \beta Z^{\Delta}(\psi(t)) \psi^{\Delta}(t)\right)\left(Z^{\beta+1}(\psi(t))\right)^{-1} .
$$

If $\beta \geqslant 1$. From $Z^{\Delta}(t)<0$, Lemmas 1 and 2 , we have

$$
\begin{aligned}
\left(Z^{\beta}(\psi(t))\right)^{\Delta} & \\
= & \beta\left\{\int_{0}^{1}[(1-h) Z(\psi(t))+h Z(\psi(\sigma(t)))]^{\beta-1} \mathrm{~d} h\right\} \\
& \times(Z(\psi(t)))^{\Delta} \\
\leqslant & \beta\left[\int_{0}^{1} Z^{\beta-1}(\psi(\sigma(t))) \mathrm{d} h\right] Z^{\Delta}(\psi(t)) \psi^{\Delta}(t) \\
= & \beta Z^{\beta-1}(\psi(\sigma(t))) Z^{\Delta}(\psi(t)) \psi^{\Delta}(t) .
\end{aligned}
$$

From (1), $\left(\mathrm{H}_{6}\right),(85)$ and $(94)$, we get

$$
\begin{gathered}
w^{\Delta}(t)=\frac{1}{Z^{\beta}(\psi(t))}\left(r(t)\left(-Z^{\Delta}(t)\right)^{\alpha-1} Z^{\Delta}(t)\right)^{\Delta} \\
-\left(r(\sigma(t))\left(-Z^{\Delta}(\sigma(t))\right)^{\alpha-1}\right. \\
\left.\quad \times Z^{\Delta}(\sigma(t))\left(Z^{\beta}(\psi(t))\right)^{\Delta}\right)
\end{gathered}
$$




$$
\begin{aligned}
& \times\left(Z^{\beta}(\psi(t)) Z^{\beta}(\psi(\sigma(t)))\right)^{-1} \\
\leqslant & -q(t) \frac{x^{\beta}(\delta(t))}{Z^{\beta}(\psi(t))} \\
- & \left(r(\sigma(t))\left(-Z^{\Delta}(\sigma(t))\right)^{\alpha-1} Z^{\Delta}(\sigma(t))\right. \\
& \left.\times \beta Z^{\Delta}(\psi(t)) \psi^{\Delta}(t)\right) \\
\times & -q\left(Z^{\beta}(\psi(t)) \frac{x^{\beta}(\delta(t))}{Z^{\beta}(\psi(t))}\right. \\
\leqslant & \left(r(\sigma(t))\left(-Z^{\Delta}(\sigma(t))\right)^{\alpha-1} Z^{\Delta}(\sigma(t))\right. \\
& \left.\times \beta Z^{\Delta}(\psi(t)) \psi^{\Delta}(t)\right)\left(Z^{\beta+1}(\psi(t))\right)^{-1} .
\end{aligned}
$$

Therefore, for $\beta>0$, from (93) and (95), we get

$$
\begin{aligned}
& w^{\Delta}(t) \\
& \leqslant-q(t) \frac{x^{\beta}(\delta(t))}{Z^{\beta}(\psi(t))} \\
& \quad-\frac{r(\sigma(t))\left(-Z^{\Delta}(\sigma(t))\right)^{\alpha-1} Z^{\Delta}(\sigma(t)) \beta Z^{\Delta}(\psi(t)) \psi^{\Delta}(t)}{Z^{\beta+1}(\psi(t))} .
\end{aligned}
$$

Noticing that $p^{\Delta}(t) \geqslant 0$ and $\tau^{\Delta}(t) \geqslant 0$, from $Z^{\Delta}(t)=x^{\Delta}(t)+$ $p^{\Delta}(t) x(\tau(t))+p(\sigma(t)) x^{\Delta}(\tau(t)) \tau^{\Delta}(t)$, we see that $x^{\Delta}(t) \leqslant 0$ for $t \geqslant T_{1}$, and from $\delta(t) \leqslant \tau(\psi(t)) \leqslant \psi(t)$ we can get

$$
\begin{aligned}
\frac{x^{\beta}(\delta(t))}{Z^{\beta}(\psi(t))} & =\left(\left(\frac{x(\psi(t))}{x(\delta(t))}+p(\psi(t)) \frac{x(\tau(\psi(t)))}{x(\delta(t))}\right)^{-1}\right)^{\beta} \\
& \geqslant\left(\frac{1}{1+p(\psi(t))}\right)^{\beta} .
\end{aligned}
$$

Thus from (86), (87), (96), (97) and the fact that $\left(r(t)\left(-Z^{\Delta}(t)\right)^{\alpha-1} Z^{\Delta}(t)\right)^{\Delta} \leqslant 0$, we have

$$
\begin{aligned}
w^{\Delta}(t) \leqslant & -\tilde{p}(t) \\
& -\frac{r(\sigma(t))\left(-Z^{\Delta}(\sigma(t))\right)^{\alpha-1} Z^{\Delta}(\sigma(t)) \beta Z^{\Delta}(t) \psi^{\Delta}(t)}{Z^{\beta+1}(\psi(t))} \\
& \times\left(\frac{r(t)}{r(\psi(t))}\right)^{1 / \alpha} \\
= & -\tilde{p}(t)-\frac{r(t)\left(-Z^{\Delta}(t)\right)^{\alpha-1} Z^{\Delta}(t) \beta Z^{\Delta}(t) \psi^{\Delta}(t)}{Z^{\beta+1}(\psi(t))} \\
& \times\left(\frac{r(t)}{r(\psi(t))}\right)^{1 / \alpha} \\
= & -\tilde{p}(t)-\frac{r(t)\left(-Z^{\Delta}(t)\right)^{\alpha-1} Z^{\Delta}(t) \beta Z^{\Delta}(t) \psi^{\Delta}(t)}{Z^{\beta+1}(\psi(t))} \\
& \times\left(\frac{r(t)}{r(\psi(t))}\right)^{1 / \alpha} \\
\leqslant & -\tilde{p}(t)-\frac{\beta M_{0}^{(\alpha-\beta) / \alpha} \psi^{\Delta}(t)}{r^{1 / \alpha}(\psi(t))}(-w(t))^{(\alpha+1) / \alpha},
\end{aligned}
$$

where $\tilde{p}(t)=q(t)(1 /(1+p(\psi(t))))^{\beta}$.

That is

$$
w^{\Delta}(t)+\tilde{p}(t)+\frac{\beta M_{0}^{(\alpha-\beta) / \alpha} \psi^{\Delta}(t)}{r^{1 / \alpha}(\psi(t))}(-w(t))^{(\alpha+1) / \alpha} \leqslant 0,
$$

$$
t \geqslant T_{1} \text {. }
$$

Multiplying (99) (with $t$ replaced by $s$ ) by $V^{\alpha}(\sigma(s)$ ), integrating it with respect to $s$ from $T_{1}$ to $t$, we have

$$
\begin{aligned}
V^{\alpha}(t) & w(t)-V^{\alpha}\left(T_{1}\right) w\left(T_{1}\right)-\int_{T_{1}}^{t}\left(V^{\alpha}(s)\right)^{\Delta} w(s) \Delta s \\
& +\int_{T_{1}}^{t} \tilde{p}(s) V^{\alpha}(\sigma(s)) \Delta s \\
& +\int_{T_{1}}^{t} \frac{\beta M_{0}^{(\alpha-\beta) / \alpha} V^{\alpha}(\sigma(s)) \psi^{\Delta}(s)}{r^{1 / \alpha}(\psi(s))}(-w(s))^{(\alpha+1) / \alpha} \Delta s \leqslant 0 .
\end{aligned}
$$


Next, we consider the following two cases.

Case (i) (let $0<\alpha<1$ ). From Lemma 2 and $V^{\Delta}(t)=$ $-r^{-1 / \alpha}(\psi(t)) \psi^{\Delta}(t)<0$, we have

$$
\begin{aligned}
\left(V^{\alpha}(t)\right)^{\Delta} & =\alpha\left\{\int_{0}^{1}[(1-h) V(t)+h V(\sigma(t))]^{\alpha-1} \mathrm{~d} h\right\} V^{\Delta}(t) \\
& \geqslant \alpha\left[\int_{0}^{1} V^{\alpha-1}(\sigma(t)) \mathrm{d} h\right] V^{\Delta}(t) \\
& =\alpha V^{\alpha-1}(\sigma(t)) V^{\Delta}(t) .
\end{aligned}
$$

From (100) and (101), we get

$$
\begin{aligned}
V^{\alpha}(t) & w(t)-V^{\alpha}\left(T_{1}\right) w\left(T_{1}\right) \\
& -\int_{T_{1}}^{t} \alpha V^{\alpha-1}(\sigma(s)) V^{\Delta}(s) w(s) \Delta s \\
& +\int_{T_{1}}^{t} \tilde{p}(s) V^{\alpha}(\sigma(s)) \Delta s \\
+ & \int_{T_{1}}^{t} \frac{\beta M_{0}^{(\alpha-\beta) / \alpha} V^{\alpha}(\sigma(s)) \psi^{\Delta}(s)}{r^{1 / \alpha}(\psi(s))}(-w(s))^{(\alpha+1) / \alpha} \Delta s \leqslant 0 .
\end{aligned}
$$

That is

$$
\begin{aligned}
V^{\alpha}(t) w(t) & +\int_{T_{1}}^{t} \tilde{p}(s) V^{\alpha}(\sigma(s)) \Delta s \\
-\int_{T_{1}}^{t} & {\left[\alpha V^{\alpha-1}(\sigma(s))\left(-V^{\Delta}(s)\right)(-w(s)) \Delta s\right.} \\
& \left.-\frac{\beta M_{0}^{(\alpha-\beta) / \alpha} V^{\alpha}(\sigma(s)) \psi^{\Delta}(s)}{r^{1 / \alpha}(\psi(s))}(-w(s))^{(\alpha+1) / \alpha}\right] \Delta s
\end{aligned}
$$$$
\leqslant V^{\alpha}\left(T_{1}\right) w\left(T_{1}\right) \text {. }
$$

Taking $a=\alpha V^{\alpha-1}(\sigma(s))\left(-V^{\Delta}(s)\right), b=\beta M_{0}^{(\alpha-\beta) / \alpha} V^{\alpha}(\sigma(s)) \psi^{\Delta}(s) /$ $r^{1 / \alpha}(\psi(s))$, by Lemma 3 and (103), we obtain

$$
\begin{aligned}
& V^{\alpha}(t) w(t)+\int_{T_{1}}^{t} \tilde{p}(s) V^{\alpha}(\sigma(s)) \Delta s \\
& \quad-\int_{T_{1}}^{t} \frac{\alpha^{\alpha} r(\psi(s))\left(\alpha V^{\alpha-1}(\sigma(s))\left(-V^{\Delta}(s)\right)\right)^{\alpha+1}}{(\alpha+1)^{\alpha+1}\left(\beta M_{0}^{(\alpha-\beta) / \alpha} V^{\alpha}(\sigma(s)) \psi^{\Delta}(s)\right)^{\alpha}} \Delta s \\
& \leqslant V^{\alpha}\left(T_{1}\right) w\left(T_{1}\right) .
\end{aligned}
$$

That is

$$
\begin{aligned}
V^{\alpha}(t) w(t) \leqslant V^{\alpha}\left(T_{1}\right) w\left(T_{1}\right) & \\
-\int_{T_{1}}^{t}\left[\tilde{p}(s) V^{\alpha}(\sigma(s))\right. & \\
& \left.\quad-\frac{\alpha^{2 \alpha+1} r^{-1 / \alpha}(\psi(s)) \psi^{\Delta}(s)}{(\alpha+1)^{\alpha+1} \beta^{\alpha} M_{0}^{\alpha-\beta} V(\sigma(s))}\right] \Delta s .
\end{aligned}
$$

By (83), we get a contradiction with (91).

Case (ii) (let $\alpha \geqslant 1$ ). From Lemma 2 and $V^{\Delta}(t)<0$, we get

$$
\begin{aligned}
\left(V^{\alpha}(t)\right)^{\Delta} & =\alpha\left\{\int_{0}^{1}[(1-h) V(t)+h V(\sigma(t))]^{\alpha-1} \mathrm{~d} h\right\} V^{\Delta}(t) \\
& \geqslant \alpha\left[\int_{0}^{1} V^{\alpha-1}(t) \mathrm{d} h\right] V^{\Delta}(t)=\alpha V^{\alpha-1}(t) V^{\Delta}(t) .
\end{aligned}
$$

From (100) and (106), we obtain

$$
\begin{aligned}
& V^{\alpha}(t) w(t)-V^{\alpha}\left(T_{1}\right) w\left(T_{1}\right)-\int_{T_{1}}^{t} \alpha V^{\alpha-1}(s) V^{\Delta}(s) w(s) \Delta s \\
& +\int_{T_{1}}^{t} \widetilde{p}(s) V^{\alpha}(\sigma(s)) \Delta s \\
& +\int_{T_{1}}^{t} \frac{\beta M_{0}^{(\alpha-\beta) / \alpha} V^{\alpha}(\sigma(s)) \psi^{\Delta}(s)}{r^{1 / \alpha}(\psi(s))}(-w(s))^{(\alpha+1) / \alpha} \Delta s \leqslant 0 .
\end{aligned}
$$

That is

$$
\begin{aligned}
& V^{\alpha}(t) w(t)+\int_{T_{1}}^{t} \tilde{p}(s) V^{\alpha}(\sigma(s)) \Delta s \\
& -\int_{T_{1}}^{t}\left[\alpha V^{\alpha-1}(s)\left(-V^{\Delta}(s)\right)(-w(s)) \Delta s\right. \\
& \left.\quad-\frac{\beta M_{0}^{(\alpha-\beta) / \alpha} V^{\alpha}(\sigma(s)) \psi^{\Delta}(s)}{r^{1 / \alpha}(\psi(s))}(-w(s))^{(\alpha+1) / \alpha}\right] \Delta s \\
& \leqslant V^{\alpha}\left(T_{1}\right) w\left(T_{1}\right) .
\end{aligned}
$$

Taking $a=\alpha V^{\alpha-1}(s)\left(-V^{\Delta}(s)\right), b=\beta M_{0}^{(\alpha-\beta) / \alpha} V^{\alpha}(\sigma(s)) \psi^{\Delta}(s) /$ $r^{1 / \alpha}(\psi(s))$, by Lemma 3 and (108), we obtain

$$
\begin{aligned}
& V^{\alpha}(t) w(t)+\int_{T_{1}}^{t} \tilde{p}(s) V^{\alpha}(\sigma(s)) \Delta s \\
& \quad-\int_{T_{1}}^{t} \frac{\alpha^{\alpha} r(\psi(s))\left(\alpha V^{\alpha-1}(s)\left(-V^{\Delta}(s)\right)\right)^{\alpha+1}}{(\alpha+1)^{\alpha+1}\left(\beta M_{0}^{(\alpha-\beta) / \alpha} V^{\alpha}(\sigma(s)) \psi^{\Delta}(s)\right)^{\alpha}} \Delta s \\
& \leqslant V^{\alpha}\left(T_{1}\right) w\left(T_{1}\right) .
\end{aligned}
$$


That is

$$
\begin{aligned}
& V^{\alpha}(t) w(t) \\
& \leqslant V^{\alpha}\left(T_{1}\right) w\left(T_{1}\right) \\
& -\int_{T_{1}}^{t}\left[\tilde{p}(s) V^{\alpha}(\sigma(s))\right. \\
& \left.\quad-\frac{\alpha^{2 \alpha+1} r^{-1 / \alpha}(\psi(s)) V^{\alpha^{2}-1}(s) \psi^{\Delta}(s)}{(\alpha+1)^{\alpha+1} \beta^{\alpha} M_{0}^{\alpha-\beta} V^{\alpha^{2}}(\sigma(s))}\right] \Delta s .
\end{aligned}
$$

By (83), we get a contradiction with (91). This completes the proof of Theorem 9.

\section{Examples}

Example 10. Consider the following dynamic equation:

$$
\begin{gathered}
{\left[\left|\left(x(t)+\frac{1}{1+t^{2}} x(\delta(t))\right)^{\Delta}\right|^{\alpha-1}\left(x(t)+\frac{1}{1+t^{2}} x(\delta(t))\right)^{\Delta}\right]^{\Delta}} \\
+\frac{1}{t^{2}}\left(1+\frac{1}{\delta^{2}(t)}\right)^{\beta}|x(\delta(t))|^{\beta-1} x(\delta(t))=0, \quad t \in \mathbb{T}
\end{gathered}
$$

where $\alpha>\beta>1$ are constants. In (111), $r(t)=1, p(t)=$ $1 /\left(1+t^{2}\right), q(t)=\left(1 / t^{2}\right)\left(1+1 / \delta^{2}(t)\right)^{\beta}$.

If $\mathbb{T}=\overline{q_{0}^{\mathbb{Z}}}=\left\{q_{0}^{n}: n \in \mathbb{Z}\right\} \cup\{0\}$, and $\delta(t)=t / q_{0}$, where $q_{0}>1$ and $q_{0} \in \mathbb{R}$, then $\delta^{\Delta}(t)=1 / q_{0}$. It is easy to get that $\bar{p}(t)=q(t)[1-p(\delta(t))]^{\beta}=1 / t^{2}$. Choosing $\xi(t)=t$, therefore,

$$
\begin{aligned}
\varlimsup_{t \rightarrow \infty} \int_{t_{0}}^{t}(\xi(s) \bar{p}(s) \\
\left.\quad-\frac{\left(\xi^{\Delta}(s)\right)^{2}(r(\sigma(s)))^{(\alpha-\beta) / \alpha}(r(\delta(s)))^{\beta / \alpha}}{4 \beta \xi(s)(\delta(s) / 2)^{\beta-1} \delta^{\Delta}(s) M^{\alpha-\beta}}\right) \Delta s \\
=\varlimsup_{t \rightarrow \infty} \int_{t_{0}}^{t}\left(\frac{1}{s}-\frac{2^{\beta-1} q_{0}^{\beta}}{4 \beta s^{\beta} M^{(\alpha-\beta) / \alpha}}\right) \Delta s=\infty .
\end{aligned}
$$

Hence, by Theorem 6, (111) is oscillatory.

Example 11. Consider the following dynamic equation:

$$
\begin{aligned}
& {\left[t^{\alpha}\left|\left(x(t)+\left(1-\frac{1}{1+t^{2}}\right) x(\delta(t))\right)^{\Delta}\right|^{\alpha-1}\right.} \\
& \left.\quad \times\left(x(t)+\left(1-\frac{1}{1+t^{2}}\right) x(\delta(t))\right)^{\Delta}\right]^{\Delta} \\
& \quad+\frac{1}{t}\left(1+\frac{1}{\delta^{2}(t)}\right)^{\beta}|x(\delta(t))|^{\beta-1} x(\delta(t))=0, \quad t \in \mathbb{T},
\end{aligned}
$$

where $\alpha>\beta>1$. In (113), $r(t)=t^{\alpha}, p(t)=1-1 /\left(1+t^{2}\right)$, $q(t)=(1 / t)\left(1+\delta^{2}(t)\right)^{\beta}$.

If $\mathbb{T}=\overline{q_{0}^{\mathbb{Z}}}=\left\{q_{0}^{n}: n \in \mathbb{Z}\right\} \cup\{0\}$, and $\delta(t)=t / q_{0}$, where $q_{0}>1$ and $q_{0} \in \mathbb{R}$, then $\delta^{\Delta}(t)=1 / q_{0}$. It is easy to get that $\bar{p}(t)=q(t)[1-p(\delta(t))]^{\beta}=1 / t$. Choosing $\xi(t)=1, H(t, s)=$ $t-s$, therefore, $(t-s)^{\Delta_{s}}=-1$,

$$
\begin{aligned}
\varlimsup_{t \rightarrow \infty} & \frac{1}{H\left(t, t_{0}\right)} \int_{t_{0}}^{t} H(t, s) \xi(s) \bar{p}(s) \Delta s \\
& =\varlimsup_{t \rightarrow \infty} \frac{1}{t-t_{0}} \int_{t_{0}}^{t}(t-s) \frac{1}{s} \Delta s \\
& =\varlimsup_{t \rightarrow \infty} \frac{t}{t-t_{0}} \cdot \frac{1}{t} \int_{t_{0}}^{t} \frac{t-s}{s} \Delta s \\
& =\infty .
\end{aligned}
$$

Hence, by Theorem 7, (111) is oscillatory.

\section{Acknowledgments}

This research was supported by the National Natural Science Foundations of China (nos. 11171178, 61104136), Natural Science Foundation of Shandong Province of China (no. ZR2010FQ002), and Foundation of Qufu Normal University (no. XJ201014).

\section{References}

[1] S. Hilger, "Analysis on measure chains-a unified approach to continuous and discrete calculus," Results in Mathematics, vol. 18, no. 1-2, pp. 18-56, 1990.

[2] R. P. Agarwal, D. O’Regan, and S. H. Saker, "Oscillation criteria for second-order nonlinear neutral delay dynamic equations," Journal of Mathematical Analysis and Applications, vol. 300, no. 1, pp. 203-217, 2004.

[3] R. P. Agarwal, M. Bohner, and S. H. Saker, "Oscillation of second order delay dynamic equations," The Canadian Applied Mathematics Quarterly, vol. 13, no. 1, pp. 1-18, 2005.

[4] S. H. Saker, R. P. Agarwal, and D. O’Regan, "Oscillation results for second-order nonlinear neutral delay dynamic equations on time scales," Applicable Analysis, vol. 86, no. 1, pp. 1-17, 2007.

[5] S. H. Saker, D. O’Regan, and R. P. Agarwal, "Oscillation theorems for second-order nonlinear neutral delay dynamic equations on time scales," Acta Mathematica Sinica, vol. 24, no. 9, pp. 1409-1432, 2008.

[6] S. Saker, "Oscillation criteria of second-order half-linear dynamic equations on time scales," Journal of Computational and Applied Mathematics, vol. 177, no. 2, pp. 375-387, 2005.

[7] S. H. Saker, "Oscillation of second-order nonlinear neutral delay dynamic equations on time scales," Journal of Computational and Applied Mathematics, vol. 187, no. 2, pp. 123-141, 2006.

[8] S. H. Saker, "Oscillation of second-order neutral delay dynamic equations of Emden-Fowler type," Dynamic Systems and Applications, vol. 15, no. 3-4, pp. 629-644, 2006.

[9] S. H. Saker, "Oscillation criteria for a certain class of secondorder neutral delay dynamic equations," Dynamics of Continuous, Discrete \& Impulsive Systems. Series B, vol. 16, no. 3, pp. 433-452, 2009. 
[10] S. H. Saker and S. R. Grace, "Oscillation criteria for quasi-linear functional dynamic equations on time scales," Mathematica Slovaca, vol. 62, no. 3, pp. 501-524, 2012.

[11] S. H. Saker and D. O’Regan, "New oscillation criteria for second-order neutral functional dynamic equations via the generalized Riccati substitution," Communications in Nonlinear Science and Numerical Simulation, vol. 16, no. 1, pp. 423-434, 2011.

[12] S. H. Saker and D. O’Regan, "New oscillation criteria for second-order neutral dynamic equations on time scales via Riccati substitution," Hiroshima Mathematical Journal, vol. 42, no. 1, pp. 77-98, 2012.

[13] S. H. Saker, "Oscillation criteria for second-order quasilinear neutral functional dynamic equation on time scales," Nonlinear Oscillations, vol. 13, no. 3, pp. 407-428, 2010.

[14] T. S. Hassan, "Oscillation criteria for half-linear dynamic equations on time scales," Journal of Mathematical Analysis and Applications, vol. 345, no. 1, pp. 176-185, 2008.

[15] L. Erbe, A. Peterson, and S. H. Saker, "Asymptotic behavior of solutions of a third-order nonlinear dynamic equation on time scales," Journal of Computational and Applied Mathematics, vol. 181, no. 1, pp. 92-102, 2005.

[16] L. Erbe, A. Peterson, and S. H. Saker, "Oscillation criteria for second-order nonlinear delay dynamic equations," Journal of Mathematical Analysis and Applications, vol. 333, no. 1, pp. 505522, 2007.

[17] L. Erbe, A. Peterson, and S. H. Saker, "Hille and Nehari type criteria for third-order dynamic equations," Journal of Mathematical Analysis and Applications, vol. 329, no. 1, pp. 112131, 2007.

[18] L. Erbe, T. S. Hassan, and A. Peterson, "Oscillation criteria for nonlinear damped dynamic equations on time scales," Applied Mathematics and Computation, vol. 203, no. 1, pp. 343-357, 2008.

[19] L. Erbe, T. S. Hassan, and A. Peterson, "Oscillation criteria for nonlinear functional neutral dynamic equations on time scales," Journal of Difference Equations and Applications, vol. 15, no. 1112, pp. 1097-1116, 2009.

[20] A. Del Medico and Q. Kong, "Kamenev-type and interval oscillation criteria for second-order linear differential equations on a measure chain," Journal of Mathematical Analysis and Applications, vol. 294, no. 2, pp. 621-643, 2004.

[21] H.-W. Wu, R.-K. Zhuang, and R. M. Mathsen, "Oscillation criteria for second-order nonlinear neutral variable delay dynamic equations," Applied Mathematics and Computation, vol. 178, no. 2, pp. 321-331, 2006.

[22] S. R. Grace, R. P. Agarwal, B. Kaymakçalan, and W. Saejie, "Oscillation theorems for second order nonlinear dynamic equations," Journal of Applied Mathematics and Computing, vol. 32, no. 1, pp. 205-218, 2010.

[23] S. R. Grace, M. Bohner, and R. P. Agarwal, "On the oscillation of second-order half-linear dynamic equations," Journal of Difference Equations and Applications, vol. 15, no. 5, pp. 451-460, 2009.

[24] Y. Şahiner, "Oscillation of second order delay differential equations on time scales," Nonlinear Analysis: Theory, Methods \& Applications, vol. 63, pp. 1073-1080, 2005.

[25] D.-X. Chen, "Oscillation of second-order Emden-Fowler neutral delay dynamic equations on time scales," Mathematical and Computer Modelling, vol. 51, no. 9-10, pp. 1221-1229, 2010.
[26] M. Bohner and A. Peterson, Dynamic Equations on Time Scales: An Introduction with Applications, Birkhäuser, Boston, Mass, USA, 2001.

[27] G. H. Hardy, J. E. Littlewood, and G. Pólya, Inequalities, Cambridge University Press, Cambridge, UK, 2nd edition, 1952. 


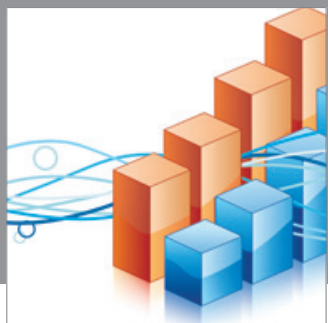

Advances in

Operations Research

mansans

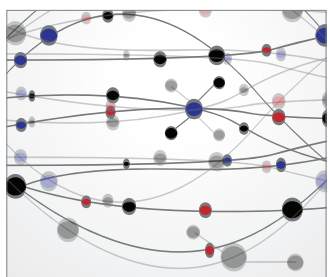

The Scientific World Journal
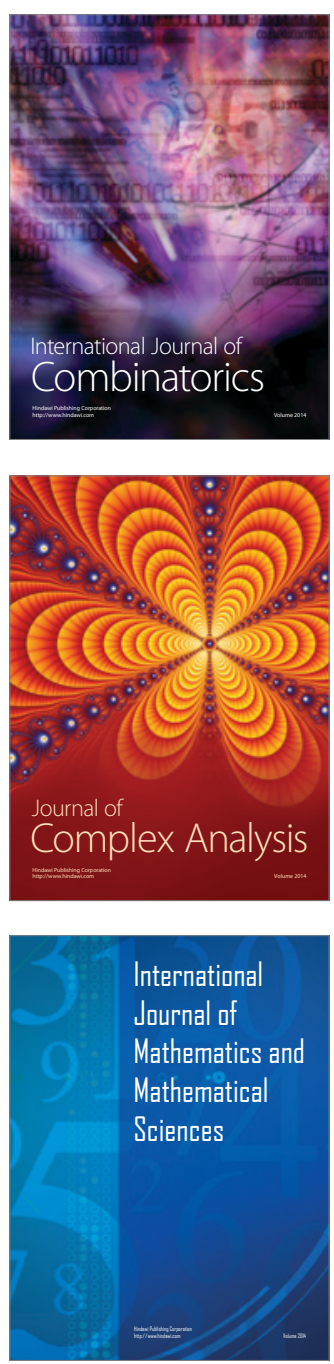
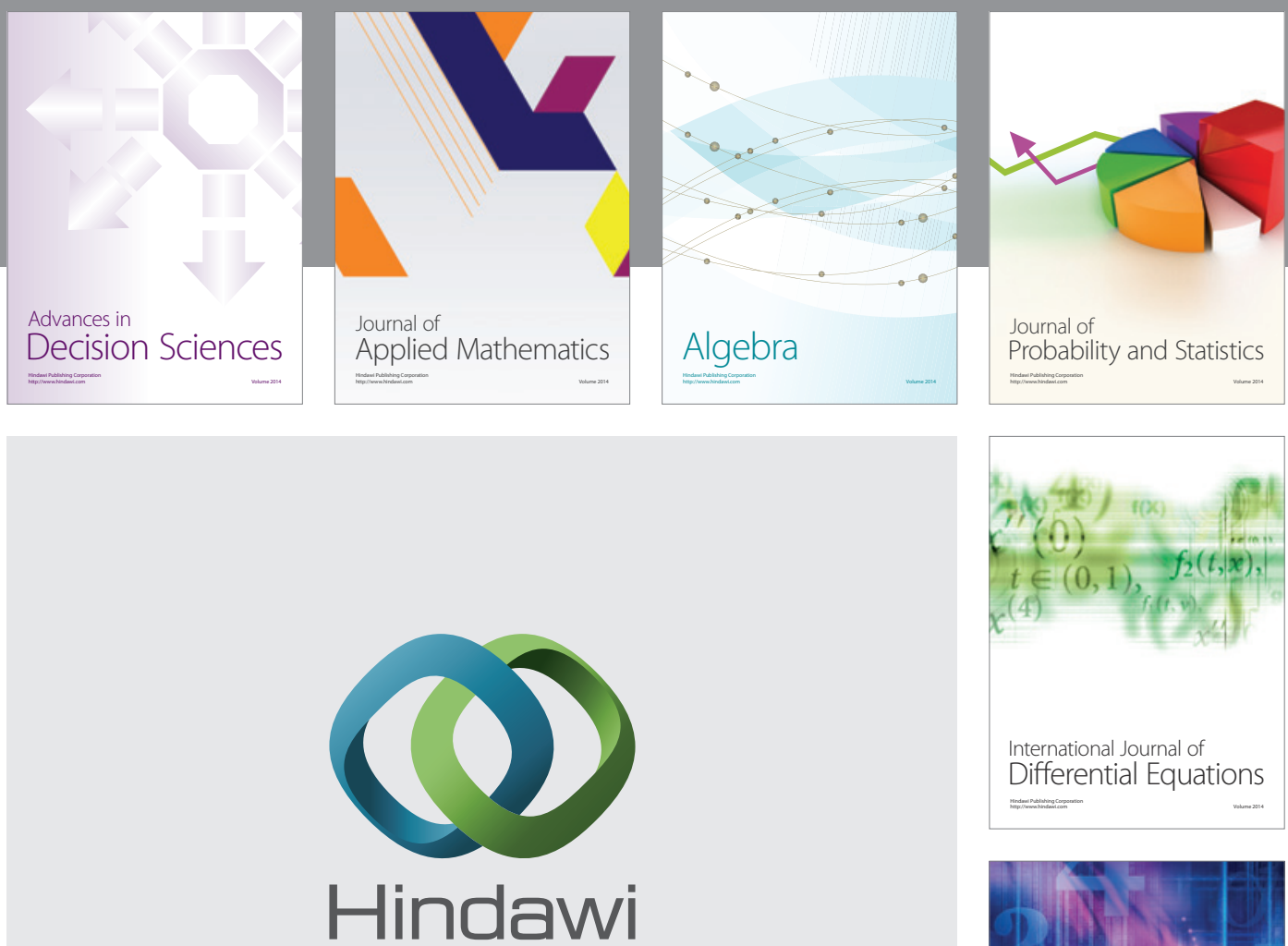

Submit your manuscripts at http://www.hindawi.com
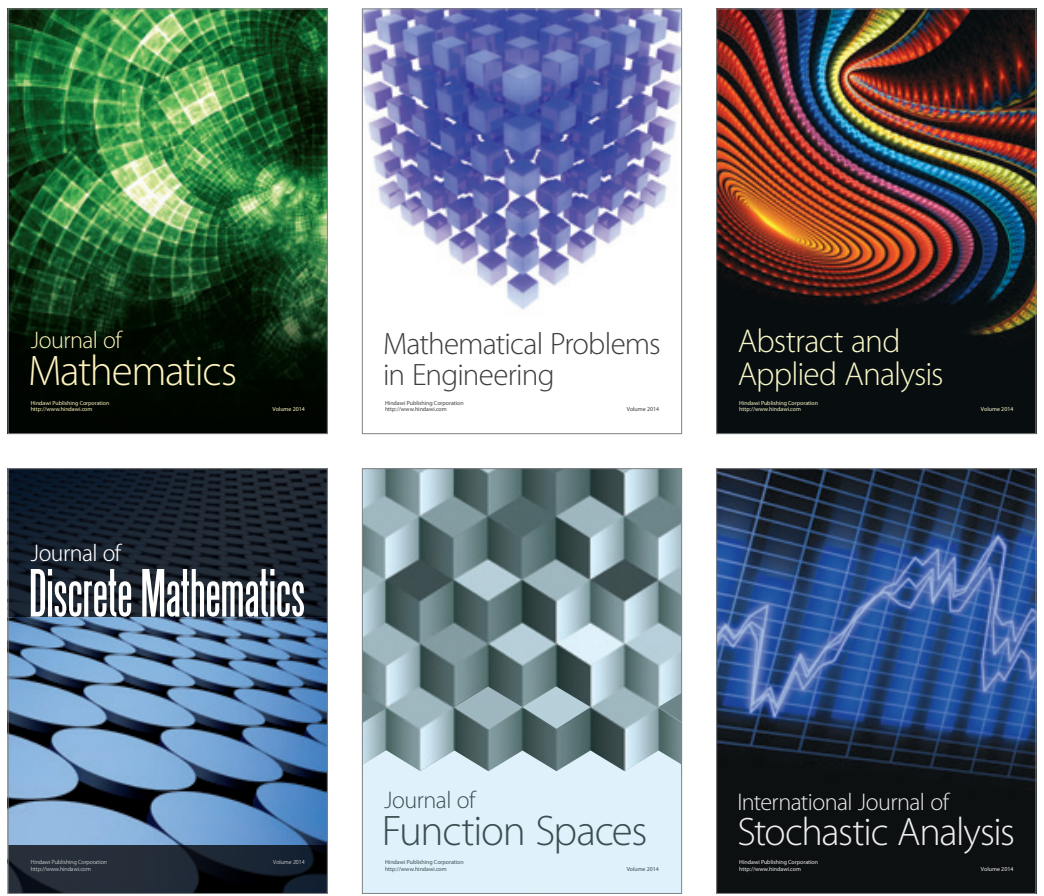

Journal of

Function Spaces

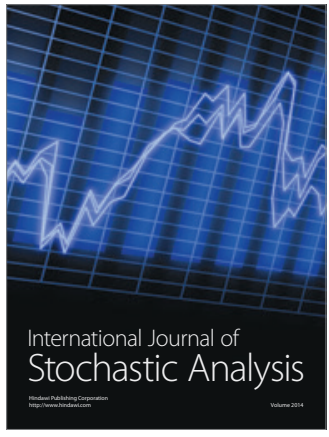

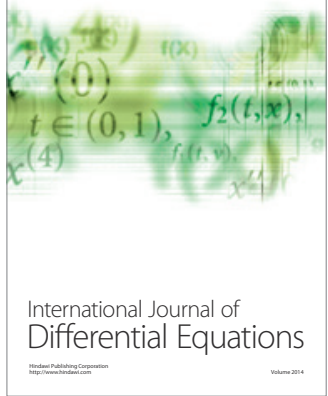
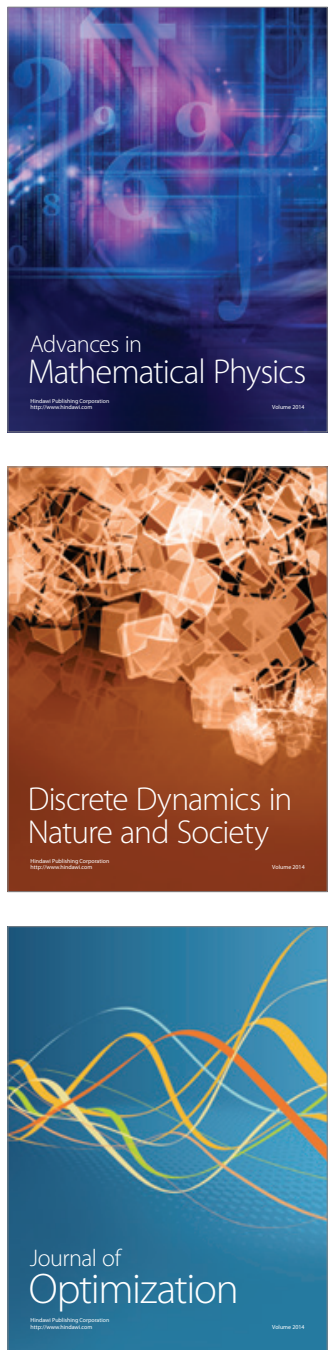